Dynamic Coefficients of Finite Length Journal Bearing. Evaluation Using a Regular Perturbation Method

Claudio E. Merelli , Daniel O. Barilá , Gustavo G. Vignolo , Lidia M. Quinzani

PII:

DOI:

S0020-7403(18)31864-2

Reference: https://doi.org/10.1016/j.jimecsci.2018.11.018 MS 4644

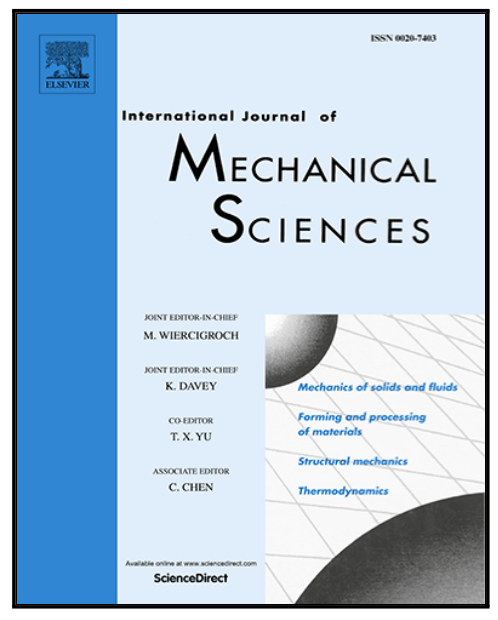

To appear in:

International Journal of Mechanical Sciences

Received date:

6 June 2018

Revised date:

23 October 2018

Accepted date:

18 November 2018

Please cite this article as: Claudio E. Merelli, Daniel O. Barilá, Gustavo G. Vignolo , Lidia M. Quinzani, Dynamic Coefficients of Finite Length Journal Bearing. Evaluation Using a Regular Perturbation Method, International Journal of Mechanical Sciences (2018), doi: https://doi.org/10.1016/j.jmecsci.2018.11.018

This is a PDF file of an unedited manuscript that has been accepted for publication. As a service to our customers we are providing this early version of the manuscript. The manuscript will undergo copyediting, typesetting, and review of the resulting proof before it is published in its final form. Please note that during the production process errors may be discovered which could affect the content, and all legal disclaimers that apply to the journal pertain. 


\section{Highlights}

- New simple analytical expressions of static and dynamic parameters are presented

- Regular perturbation method allows to extend the Ocvirk solution up to L/D and $\eta \sim 3 / 4$

- Force, friction factor, lubricant flow rate and attitude angle are considered

- Damping and stiffness coefficients are predicted as a function of L/D and $\eta$

- The proposed method stands as a powerful tool to use in design and analysis of HJBs

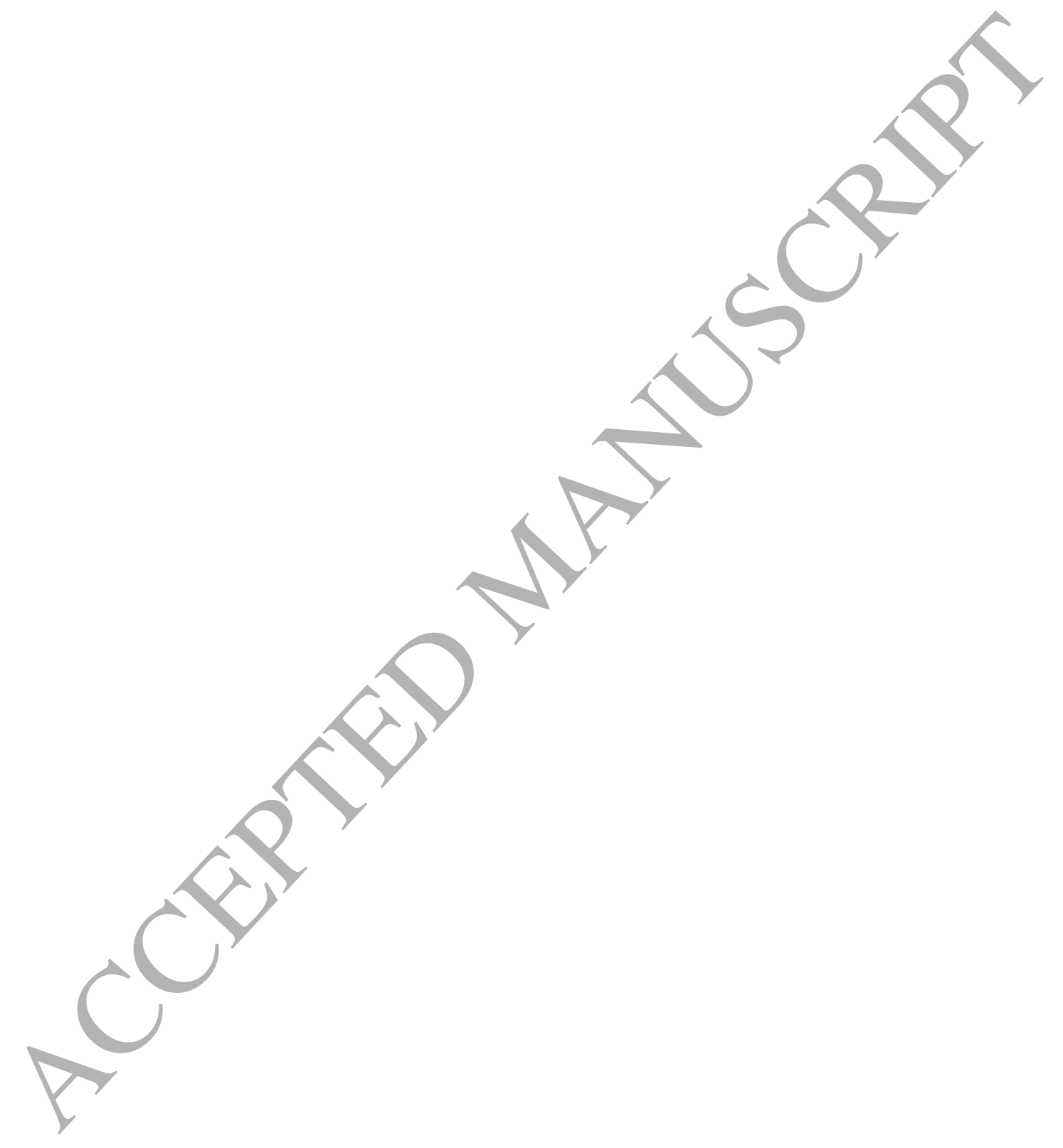




\title{
Dynamic Coefficients of Finite Length Journal Bearing. Evaluation Using \\ a Regular Perturbation Method
}

\author{
Claudio E. Merelli ${ }^{1,2,3,}$, Daniel O. Barilá ${ }^{2}$, Gustavo G. Vignolo ${ }^{2}$ and \\ Lidia M. Quinzani ${ }^{3}$ \\ ${ }^{1}$ Departamento de Ingeniería, Universidad Nacional del Sur (UNS), \\ Av. Alem 1253 - (8000) Bahía Blanca, ARGENTINA
}

${ }^{2}$ LMF, Facultad de Ingeniería, Universidad Nacional de la Patagonia San Juan Bosco, Ruta Prov. No 1 km 4 - (9000) Comodoro Rivadavia, ARGENTINA

${ }^{3}$ Planta Piloto de Ingeniería Química (PLAPIQUI), Dpto. de Ingeniería Química, UNS- CONICET - Camino La Carrindanga km 7 - (8000) Bahía Blanca, ARGENTINA

* Corresponding author: cemerelli@plapiqui.edu.ar - FAX (54)291-4861600

Declarations of interest: none

Keywords: Dynamic coefficients; Journal bearing; Perturbation method

\begin{abstract}
A set of simple expressions is deduced for static and dynamic parameters associated to hydrodynamic journal bearings (JB). The behavior of this system is governed by two dimensionless numbers, the aspect ratio, $L / D$, and the eccentricity ratio, $\eta$. In a previous work, we presented a regular perturbation method that extended the Ocvirk solution and successfully described isothermal JBs up to $L / D$ and $\eta$ of $\sim 1 / 2$. Presently, we extend that methodology, modified using a smaller perturbation parameter, to obtain analytical expressions of the dynamic coefficients, as well as static variables like friction factor, load carrying capacity, lubricant flow rate and phase angle. The deduced expressions successfully describe the static and dynamic behavior of JBs up to $L / D$ and $\eta$ of $\sim 3 / 4$.
\end{abstract}




\section{INTRODUCTION}

Fluid film lubrication occurs when opposing bearing surfaces are completely separated by a lubricant film. Hydrodynamic lubrication is described by the system of differential equations corresponding to mass, energy and momentum balances applied to the thin fluid film between journal and bearing walls in relative motion. In the particular case of a Newtonian fluid and slow, laminar, incompressible and isothermal flow, the mass and momentum equations can be simplified and then integrated into the film thickness to produce the so called Reynolds Equation [1-3]. This partial differential equation describes the pressure distribution in the thin fluid film with apparent simplicity.

An order of magnitude analysis of the equations that describe the flow in hydrodynamic journal bearings (JB) shows that the flow is largely affected by the value of two geometric relations, the square of the aspect ratio (length over diameter), $(L / D)^{2}$, and the eccentricity ratio, $\eta$, defined as eccentricity (distance between rotor and bearing loci) over clearance (difference between bearing and rotor radius). The Reynolds Equation has analytical solution in the limit of both, $L / D \rightarrow 0$ and $L / D \rightarrow \infty$. They are known, respectively, as the infinitely short (ISJB) and infinitely long (ILJB) journal bearing approximations, and as the Ocvirk and Sommerfeld solutions as well. In the case of JBs of finite length, an exact analytical solution of the Reynolds Equation has not yet been found [2]. In fact, in a previous publication, we presented an analytical approximate solution of the Reynolds equation for isothermal finite length JBs by means of the regular perturbation method, using $(L / D)^{2}$ as the perturbation parameter [4]. The novelty of that method lays on the treatment of the Ocvirk number as an expansible parameter. The first-order solution obtained with the proposed method extends the description of the Ocvirk solution (which describes reasonably well the behavior of finite length JBs up to $L / D \sim 1 / 4$ and relatively small eccentricities) up to $L / D \sim 1 / 2$ and $\eta \sim 1 / 2$ (and combinations of larger eccentricities with smaller aspect ratios, or vice versa). Presently, we extend that methodology, modified using a smaller perturbation parameter, to obtain analytical expressions of more static variables and, what is more important, the dynamic coefficients associated to hydrodynamic JBs.

Hydrodynamic JBs are considered a vital component of all rotating machinery. They are mainly used to support radial loads under high speed operating conditions. One of the essential aspects of JBs is their dynamic characteristics [1-3]. The stiffness and damping behavior of this mechanism affects its critical speed and, consequently, the stability of the rotor. This is of great importance especially in applications where the bearing is used as a 
shock absorber or to dissipate destabilizing effects [3]. There are many works in the literature that deal with the calculation of the dynamic characteristics of JBs of different configurations, even considering additional circumstances such as misalignment of the shaft or rotor flexibility [5-16, and references within]. Most of these authors have used numerical methods to solve the Reynolds equation and calculate the dynamic coefficients, and a few have considered empirical or approximate analytical methods with different degrees of difficulty. For example, Yuan and Di-Gong [14] analyze the effects of partial-grooving on the performance of spiral-grooved spherical and conical bearings using a perturbation method to determine the dynamic coefficients. Analytical but very complex expressions of the coefficients are obtained after considering first order perturbed expressions of the film thickness and the pressure using the eccentricity as the perturbation parameter. Rao and coworkers [6] extended the approach of Reason and Narang [17] and determined the dynamic coefficients in finite length JBs combining the static and dynamic pressure fields of the limit cases of ISJB and ILJB. Interestingly, this empirical method, in which the limit solutions are combined using the harmonic pressure average technique, successfully predicts the coefficients for a wide range of eccentricities and aspect ratios. On the other hand, Chasalevris and Sfyris $[9,18]$ proposed a complex but more robust methodology to determine analytical expressions of the dynamic coefficients. They solve the Reynolds equation considering separation of variables in additive and multiplicative form extending the description of the Sommerfeld solution (ILJB). The procedure gives place to four particular solutions of the Reynolds equation, which add to that of the homogenous one. The SturmLiouville problems generated have direct forms of solution except in one case, which is solved using a power series. The dynamic coefficients, as well as the static parameters, so calculated agree with exact numerical results in the case of long JBs $(L / D=4)$ and become further apart as the aspect ratio decreases (mainly for $L / D<1$ ).

The dynamic coefficients have also been determined experimentally in several works [19$24]$ and compared and commented in some reviews [25,26]. Analyzing those works, it can be appreciated that the experimental results have been generally produced to evaluate the performance of a particular configuration and their applicability to generic rotors is quite limited. However, experimental validation of the bearing models is very important. Both, data and model predictions of stiffness, damping, and mass coefficients are sensitive to design parameters, such as bearing dimensions, manufacturing tolerances, method of exciting the rotor-bearing system, thermal effects and elastic deformation, and change in fluid parameters 
and flow conditions [26]. In the case of experimental results, the quality of the data is also related to the filtering and conditioning techniques. For all these reasons, agreement between theory and experiment with respect to dynamic bearing coefficients is seldom better than 10$20 \%$, even when using numerical solution of the most realistic models [26]. In an early work, Glienicke [19] assembled one of the first reliable systems that allowed the determination of the dynamic coefficients of JBs. He considered four characteristic turbine bearings with $L / D=0.5$, and then applied the obtained results to predict weak conditions of instability. After that, various and different systems have been assembled and tested. One of the most popular methods for experimentally measuring the frequency response functions of rotor bearing systems is to perturb the system using a sinusoidal force input. For example, Zhao and coworkers [21] used this method to analyze the dynamic behavior of plain-cylinder JBs in one of the latest studies in this area.

In this paper, we extend the use of the regular perturbation method previously proposed [4] to calculate analytical expressions of the dynamic coefficients for isothermal finite length JBs, as well as some static parameters, like phase angle and lubricant flow rate, which were not included in the original work. New more simplified expressions of the static pressure, Ocvirk number, and friction coefficient are also included.

\section{GOVERNING EQUATIONS}

Figure 1 shows the system under study. It consists of a journal of radius $R$ rotating at an angular velocity $\omega$ within a static bearing of radius $R_{\mathrm{B}}$ and length $L$. A liquid lubricant fluid fills the gap between them. The action of a load $W$ displaces the rotor a distance $e$ (eccentricity) respect to the bearing center, generating a fluid film of variable height, $H(\theta)$. Under high speed operating conditions, the pressure field generated in the fluid layer gives place to a reaction force $F$ that withstands the load with low friction. The combined effect of rotation and loading produces the so called "attitude angle", $\phi$, that is, an angle between the line of centers and the line of action of the applied static load. The mathematical analysis of the flow can be performed in Cartesian coordinates (see Figure 1), neglecting the effects of curvature, since the clearance, $c_{R}=R_{\mathrm{B}}-R$, is much smaller than $R$. The gap $H(\theta)$ can then be calculated from trigonometric relationships, resulting in:

$$
H=c_{R}+R+e \cos (\theta)-R \sqrt{1-\left(\frac{e}{R}\right)^{2} \sin ^{2}(\theta)} \approx c_{R}+e \cos (\theta)
$$


which defines

$$
h=\frac{H}{c_{R}}=1+\frac{e}{c_{R}} \cos (\theta)=1+\eta \cos (\pi \Theta)
$$

where $\eta=\frac{e}{c_{R}}$ is the eccentricity ratio.
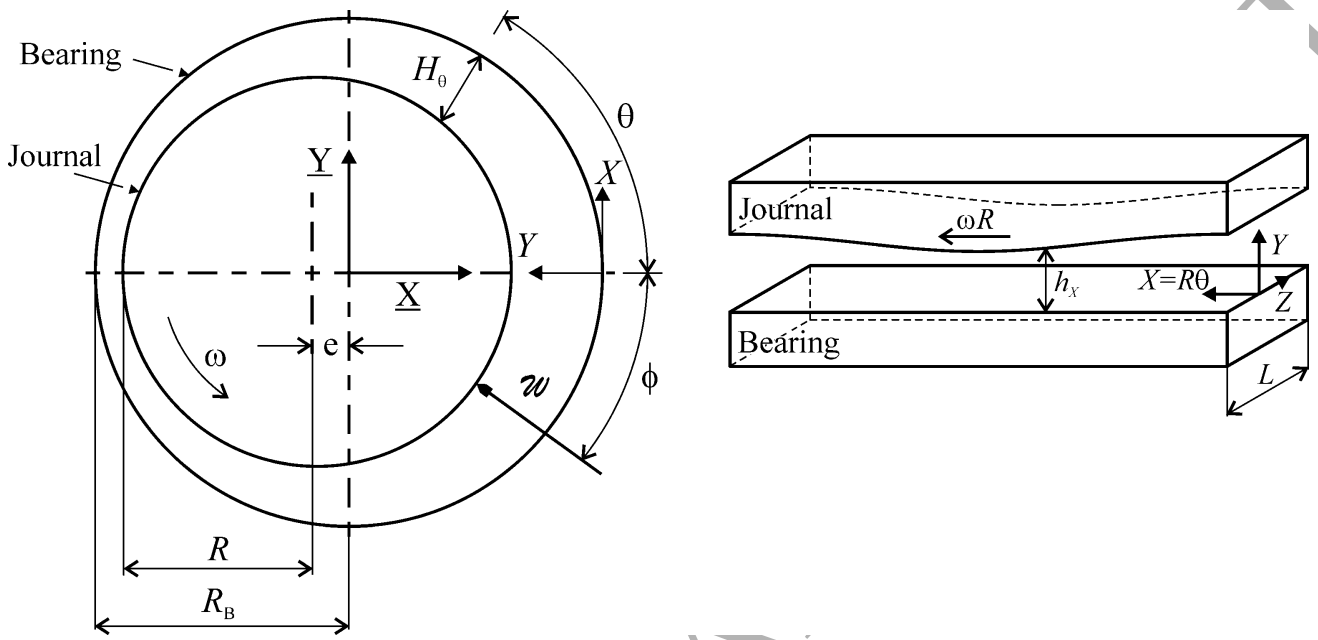

Figure 1. Geometry notation and coordinate systems (left). Representation in Cartesian coordinates (right).

Under the listed conditions, the mass and momentum balances that describe the flow of an incompressible Newtonian liquid under negligible gravitational effects and isothermal laminar flow with Reynolds number ( $\left.\operatorname{Re}=\frac{\rho U R}{\mu}\right)$ of order one or smaller, are:

$$
\begin{aligned}
& 0=\frac{1}{\pi} \frac{\partial u}{\partial \Theta}+\frac{\partial v}{\partial y}+\frac{\partial w}{\partial z} \\
& 0=-\frac{1}{\pi}\left(\frac{L}{R}\right)^{2} \frac{\partial p^{\prime}}{\partial \Theta}+\frac{\partial^{2} u}{\partial y^{2}} \\
& 0=-\frac{\partial p^{\prime}}{\partial y}+\left(\frac{c}{L}\right)^{2} \frac{\partial^{2} v}{\partial y^{2}} \\
& 0=-\frac{\partial p^{\prime}}{\partial z}+\frac{\partial^{2} w}{\partial y^{2}}
\end{aligned}
$$


where the dimensionless variables are:

$$
\Theta=\frac{X}{\pi R}, y=\frac{Y}{c_{R}}, z=\frac{Z}{L}, u=\frac{V_{X}}{U}, v=\frac{V_{Y}}{V}, w=\frac{V_{Z}}{W}, p^{\prime}=\frac{P-P_{E X T}}{P_{R E F}}
$$

The parameters $U, V$ and $W$ are the characteristic values of the longitudinal $\left(V_{X}\right)$, transversal $\left(V_{Y}\right)$ and axial $\left(V_{Z}\right)$ velocities, respectively. $P_{\text {EXT }}$ is the ambient pressure, external to the lubricant, and $P_{\mathrm{REF}}$ is the reference pressure. Equations (3) to (6) also consider that $K=\frac{U c_{R}}{R}$ and $W=\frac{U R}{L}$, which come from the assumption that all terms in the mass balance have similar order of magnitude. $P_{\mathrm{REF}}$ is taken as the order of magnitude of the pressure in the ISJB approximation $[3,4]$,

$$
P_{R E F}=\frac{\mu U}{R}\left(\frac{R}{c_{R}}\right)^{2}\left(\frac{L}{R}\right)^{2}
$$

According to Eq. (5), the pressure gradient in the transversal direction (y) is negligible, allowing for Eqs. (4) and (6) to be integrated in this direction. The calculated velocities $u$ and $w$ can then be replaced into the mass balance, Eq. (3), to give place (after integration in the $y$ direction and some solving) to the Reynolds equation [1-4]:

$$
\frac{d h}{d t}+\frac{1}{2 \pi} \frac{d h}{d \Theta}=\frac{1}{12 \pi^{2}}\left(\frac{L}{R}\right)^{2} \frac{\partial}{\partial \Theta}\left(h^{3} \frac{\partial p^{\prime}}{\partial \Theta}\right)+\frac{1}{12} \frac{\partial}{\partial z}\left(\frac{\partial p^{\prime}}{\partial z} h^{3}\right)
$$

The boundary conditions used to obtain this equation are:

$$
\left.u\right|_{y=1}=1 ;\left.u\right|_{y=0}=0 ;\left.v\right|_{y=1}=\frac{d h}{d t} ;\left.v\right|_{y=0}=0 ;\left.w\right|_{y=1}=0 ;\left.w\right|_{y=0}=0
$$

The variable $t$ that appears in Eq. (3) is a dimensionless time, $t=\frac{T}{T_{c}}$, where the value of the characteristic time, $T_{c}$, can be obtained from the boundary condition $\left.v\right|_{y=1}=\frac{d h}{d t}$, as:

$$
\left.\frac{R}{U c_{R}} V_{y}\right|_{y=1}=\frac{T_{c}}{c_{R}} \frac{d H}{d T} \rightarrow T_{c}=\frac{R}{U}
$$

Traditionally, the performance of hydrodynamic JBs is related to the "Sommerfeld number", $S$, or its equivalent for short bearings, the "Ocvirk number", $O[2,4,23]$. These numbers measure the relative importance of the pressure to the mean pressure, $P_{\mathrm{p}}$, which is 
calculated as the force acting on the shaft divided by its projected area, $P_{p}=\frac{F}{2 R L}$. In this paper we use:

$O=S\left(\frac{L}{R}\right)^{2}=\frac{\mu U}{R P_{p}}\left(\frac{R}{c_{R}}\right)^{2}\left(\frac{L}{R}\right)^{2}$

A small value of these numbers not only indicates a large load capacity, $F$, for a given $P_{\mathrm{REF}}$, but also suggests a large eccentricity. If the Ocvirk number is introduced in the Reynolds equation, its expression becomes:

$$
O\left(\frac{d h}{d t}+\frac{1}{2 \pi} \frac{d h}{d \Theta}\right)=\frac{1}{12 \pi^{2}}\left(\frac{L}{R}\right)^{2} \frac{\partial}{\partial \Theta}\left(h^{3} \frac{\partial p}{\partial \Theta}\right)+\frac{1}{12} h^{3} \frac{\partial^{2} p}{\partial z^{2}}
$$

where the new dimensionless pressure is defined as $p=\frac{P-P_{E X T}}{P_{p}}$

\section{DYNAMIC COEFFICIENTS}

The behavior of rotors is strongly influenced by the characteristics of their supports. In particular, the forces generated on the journal by the lubricant film of its bearings are nonlinear functions of the position and velocity of the center [3,24,27]. As commented above, the direction of the external load acting on the shaft does not match the direction of the centerline of journal and bearing loci. For that reason, a vibration or small change in the magnitude of the force will produce a small non-collinear displacement of the rotor with a corresponding squeeze film pressure that is generated in addition to the wedge film pressure. The extra pressure gives rise to spring and damping forces in the lubricant film influencing the stability of the rotor-bearing system [24]. Figure 2 presents the JB coordinate system and the notation associated to the displacement of the journal center in dimensionless form. This center vibrates with amplitudes $\Delta \eta(t)=\Delta e(t) / c_{\mathrm{R}}$ and $\eta_{\mathrm{s}} \Delta \psi(t)=\mathrm{e}_{\mathrm{s}} \Delta \phi(t) / 2 \pi c_{\mathrm{R}}$ around the equilibrium position $\left\{\eta_{\mathrm{s}}, \psi_{\mathrm{s}}\right\}$, being the magnitude of the displacement much smaller than $\eta_{\mathrm{s}}$. Accordingly, the lubricant film thickness changes in time, $h(\Theta, t)$, oscillating around the static position (from now on identified as $h_{s}(\Theta)$ ). The film thickness can then be expressed as the sum of the static term and a dynamic one, $h_{d}(\Theta, \mathrm{t})$ as: 


$$
\begin{aligned}
h(\Theta, t) & =h_{s}(\Theta)+h_{d}(\Theta, t) \\
\text { with } & \left\{\begin{array}{l}
h_{s}(\Theta)=1+\eta_{s} \cos (\pi \Theta) \\
h_{d}(\Theta, t)=\Delta \eta(t) \cos (\pi \Theta)+\eta_{s} \Delta \psi(t) \sin (\pi \Theta)
\end{array}\right.
\end{aligned}
$$
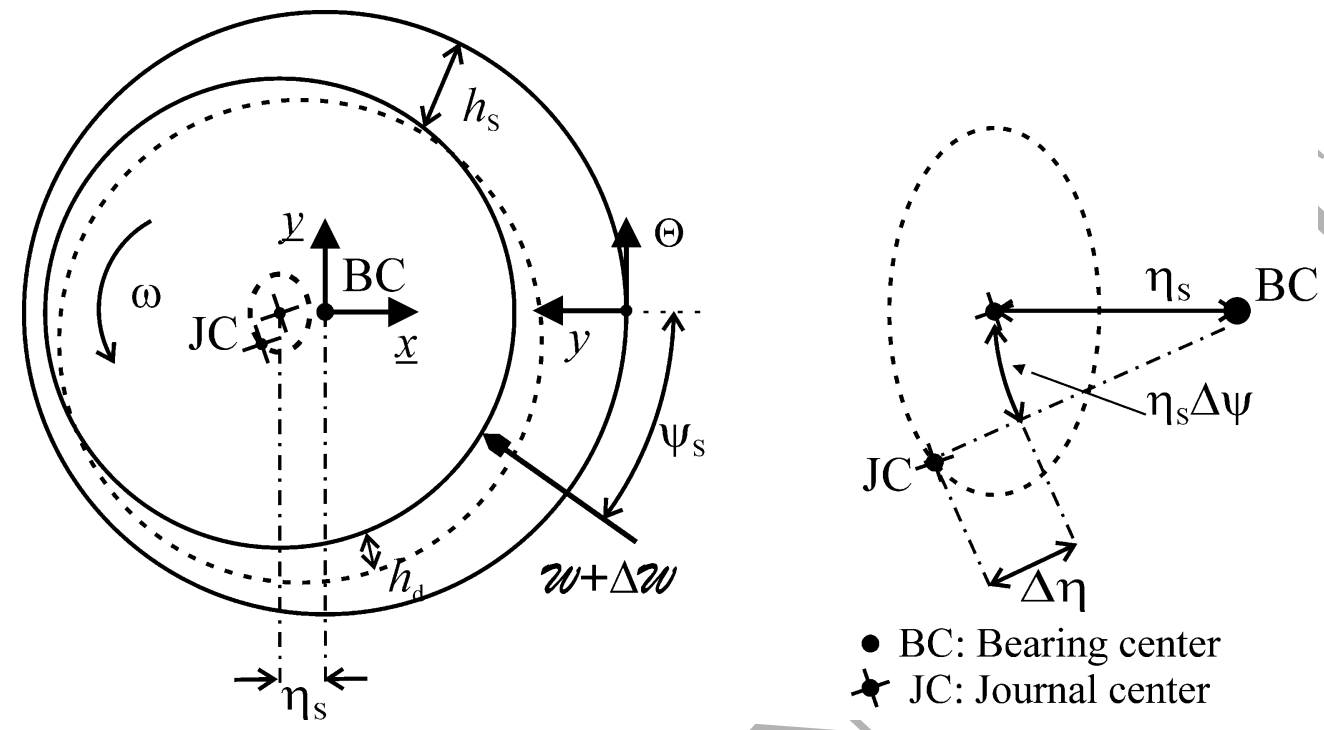

Figure 2. Representation, using dimensionless variables, of the location of bearing and journal centers when vibrating in the vicinity of the static equilibrium point.

Consequently, the dimensionless velocity and acceleration of the journal center are:

$$
\frac{d y}{d t}=\Delta \dot{\eta}(t) ; \frac{d \Theta}{d t}=\eta_{s} \Delta \dot{\psi}(t) ; \frac{d^{2} y}{d t^{2}}=\Delta \ddot{\eta}(t) ; \quad \frac{d^{2} \Theta}{d t^{2}}=\eta_{s} \Delta \ddot{\psi}(t),
$$

and the components of the dimensionless fluid film force can then be expressed as:

$$
f_{\Theta}=f_{\Theta}(\Theta, y, \dot{\Theta}, \dot{y}, \ddot{\Theta}, \ddot{y}) \quad f_{y}=f_{y}(\Theta, y, \dot{\Theta}, \dot{y}, \ddot{\Theta}, \ddot{y})
$$

through equations that, for small amplitude motions about the equilibrium position, can be linearized using Taylor series expansion around the static journal position $\left\{\eta_{s}, \psi_{s}\right\}$. That is:

$$
f_{i}=f_{i s}+\left.\frac{d f_{i}}{d \Theta}\right|_{s} \Delta \Theta+\left.\frac{d f_{i}}{d y}\right|_{s} \Delta y+\left.\frac{d f_{i}}{d \dot{\Theta}}\right|_{s} \Delta \dot{\Theta}+\left.\frac{d f_{i}}{d \dot{y}}\right|_{s} \Delta \dot{y}+\left.\frac{d f_{i}}{d \ddot{\Theta}}\right|_{s} \Delta \ddot{\Theta}+\left.\frac{d f_{i}}{d \ddot{y}}\right|_{s} \Delta \ddot{y} \quad \text { with } i=\Theta, y
$$

From these equations, the linear stiffness, damping and inertia coefficients are defined $[28,29]$, respectively, as: 
$k_{i j}=-\left.\frac{d f_{i}}{d j}\right|_{s} \quad c_{i j}=-\left.\frac{d f_{i}}{d\left(\frac{d j}{d t}\right)}\right|_{s} \quad m_{i j}=-\left.\frac{d f_{i}}{d\left(\frac{d}{d t}\left(\frac{d j}{d t}\right)\right)}\right|_{s} \quad$ with $\left\{\begin{array}{l}i=\Theta, y \\ j=\Theta, y\end{array}\right.$

where $\frac{\partial j}{\partial t}$ represents the dimensionless velocity components of the shaft center $(\dot{\Theta}$ and $\dot{y})$, and $\frac{\partial}{\partial t}\left(\frac{\partial j}{\partial t}\right)$ its accelerations ( $\ddot{\Theta}$ and $\ddot{y}$ ). Thus, for example, $k_{\Theta \mathrm{y}}$ is the dimensionless stiffness coefficient that occurs in the $\Theta$-direction due to static displacement in the $y$-direction. This cross-coupling behavior is easily demonstrated in the static calculation, where the center line has a dimensionless phase angle $(\psi)$ with respect to the applied load, which varies with eccentricity ratio. Negative signs are used in the coefficients so positive values correspond to a reaction force. The inertia coefficients $m_{\mathrm{ij}}$ will be not considered further since they become relevant for excitations at high frequencies, which are beyond the scope of this publication.

Given the definitions in Eq. (18), the dimensional stiffness and damping coefficients are:

$$
K_{i j}=\frac{2 R L P_{p}}{c_{R}} k_{i j} \quad C_{i j}=\frac{2 R^{2} L P_{p}}{U c_{R}} c_{i j}
$$

where the letters $X$ and $Y$ are used to designate the subscripts of the dimensional coefficients $K_{\mathrm{ij}}$ and $C_{\mathrm{ij}}$, instead of $\Theta$ and $y$, following the nomenclature defined in Eq. (7). Additionally, considering Eq. (12), the order of magnitude of the fluid force and the dynamic coefficients are deduced to be:

$$
\mathcal{O}(F)=\pi \mu U L \frac{4}{O}\left(\frac{R}{c_{R}}\right)^{2}\left(\frac{L}{D}\right)^{2} ; \mathcal{O}(K)=\frac{\pi \mu U L}{c_{R}} \frac{4}{O}\left(\frac{R}{c_{R}}\right)^{2}\left(\frac{L}{D}\right)^{2} ; \mathcal{O}(C)=\pi \mu L \frac{4}{O}\left(\frac{R}{c_{R}}\right)^{3}\left(\frac{L}{D}\right)^{2}
$$

The dynamic characteristics of the "journal-fluid-bearing" tribological system are frequently linked to the behavior of a mechanical model consisting in springs and dashpots [13,29]. According to this model, the system would be described by eight coefficients: four for elasticity or stiffness $\left(k_{\mathrm{ij}}\right)$ corresponding to direct $\left(k_{\Theta \Theta}, k_{\mathrm{yy}}\right)$ and cross-coupling $\left(k_{\Theta \mathrm{y}}, k_{\mathrm{y} \Theta},\right)$ springs, and four for damping $\left(c_{\mathrm{ij}}\right)$ associated to direct $\left(c_{\Theta \Theta}, c_{\mathrm{yy}}\right)$ and cross-coupling $\left(c_{\Theta \mathrm{y}}, c_{\mathrm{y} \Theta}\right)$ dashpots (see representation in Figure 3). The "direct elasticity and damping" coefficients, $k_{\Theta \Theta}, k_{\mathrm{yy}}, c_{\Theta \Theta}$ and $c_{\mathrm{yy}}$, correspond to forces directly opposed to the displacement and velocity of the shaft within the bearing. Likewise, the so-called "cross-coupling stiffness and damping" coefficients, $k_{\Theta \mathrm{y}}, k_{\mathrm{y} \Theta}, c_{\Theta \mathrm{y}}$ and $c_{\mathrm{y} \Theta}$, can be associated to displacements and velocities 
perpendicular to load application.

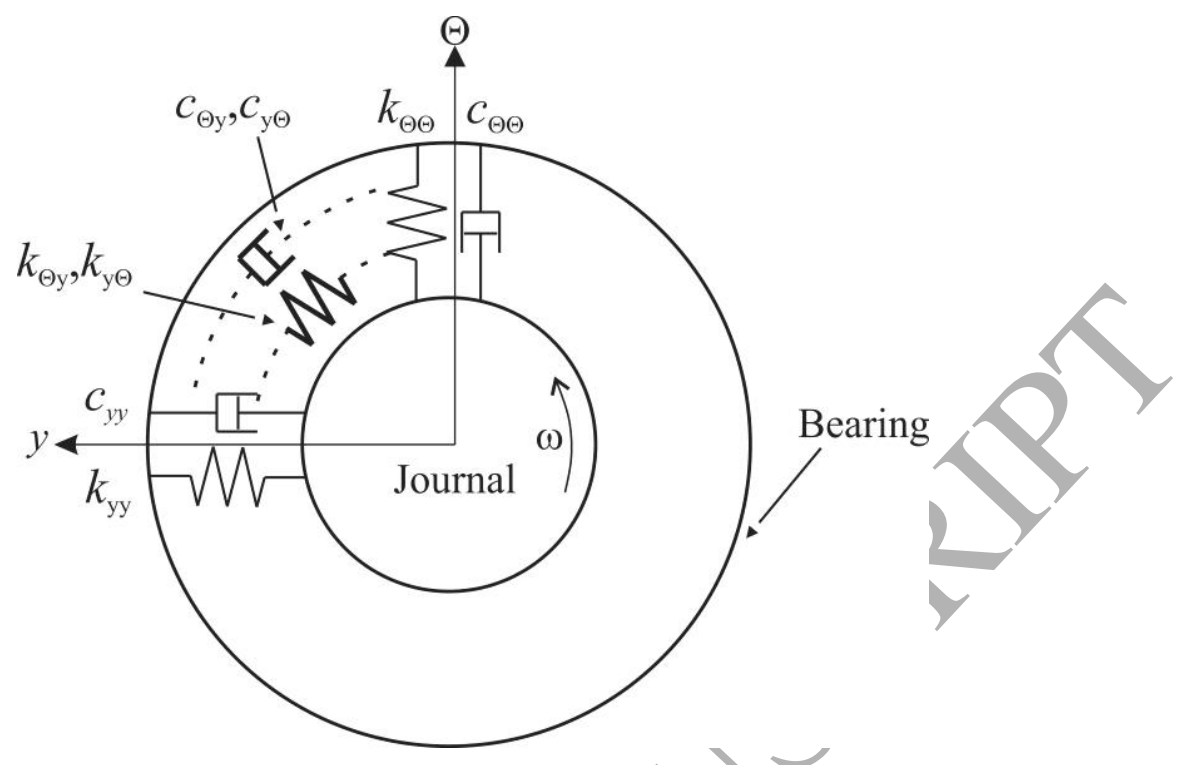

Figure 3. Schematic representation of the fluid-film dynamic coefficients according to a mechanical model of springs and dashpots.

By definition, each coefficient is evaluated in the equilibrium position with just one displacement or velocity different from zero. Consequently, the dimensionless pressure should be computed as [27,30,31]:

$$
p=p_{s}+\Delta p=p_{s}+p_{\Theta}+p_{y}+p_{\Theta}+p_{y}
$$

where $p_{\mathrm{s}}$ is the static pressure and $p_{\mathrm{j}}$ are the independent contributions from the small changes in the film thickness. Accordingly, five differential equations can be deduced, one for each contribution to $p$, by replacing $p$ and $h$ in the Reynolds equation using Eqs. (14) and (21). This step also involves/performing a first-order Taylor series expansion of the term $1 / h^{3}$ that appears in the expression of the fluid force, retaining just the first-order terms. The differential equations so obtained are listed in Appendix A. This system of equations can be solved/numerically for a given aspect ratio and eccentricity to obtain the five pressure profiles. The dynamic coefficients can then be calculated from these profiles, previous integration to evaluate the fluid film force, as it will be shown in next Section. 


\section{PROPOSED SOLUTION}

As commented in the Introduction, most researchers use numerical methods to calculate the dynamic coefficients, and just a few have considered empirical or approximate analytical methods $[6,9,14]$. Given the success of the regular perturbation method previously proposed to solve the Reynolds equation under static conditions [4], that methodology is extended to calculate analytical expressions of the dynamic coefficients for isothermal finite length JBs. The flow rate and attitude or phase angle $(\phi)$, which were not included in the previous work, are also calculated.

The proposed methodology considers the expansion of both, pressure and Ocvirk number, using a perturbation parameter, $\varepsilon$, based in the geometric ratio length over diameter [4]. In fact, the perturbation parameter used originally is $(L / D)^{2}$. However, here we propose the use of the half-length of the bearing $(L / 2)$ in the calculation of $\varepsilon$, resulting in $\varepsilon=(L / 2 D)^{2}$. This selection of perturbation parameter is actually more representative of the system (given its symmetry about $z=0$ ) and it allows to increase the range of aspect ratios where the approximation may be used (keeping $\varepsilon<1$ ).

All contributions to the pressure (static and dynamic ones) and the Ocvirk number are then expanded using $\varepsilon=(L / 2 D)^{2}$, that is

$$
\begin{aligned}
& p_{i}=p_{i 0}+\varepsilon p_{i 1}+\mathcal{O}\left(\varepsilon^{2}\right) \quad \text { with } i=s, \Theta, y, \dot{\Theta}, \dot{y} \\
& O=O_{0}+\varepsilon O_{1}+\mathcal{O}\left(\varepsilon^{2}\right)
\end{aligned}
$$

The pressure profiles can then be obtained by introducing these expansions into the equations in Appendix A and solving keeping just the terms up to order $\varepsilon$. Their expressions are listed in the Appendix B. As expected, the five $p_{\mathrm{i} 0}$ depend just on $O_{0}$ while the $p_{\mathrm{i} 1}$ are function of both, $O_{0}$ and $O_{1}$,

The calculation of the zero- and first-order Ocvirk numbers involves computing the mean pressure $P_{p}=\frac{F}{2 R L}$, where the load capacity, $F$, is:

$$
F^{2}=F_{X}{ }^{2}+F_{Y}^{2}=\left[\int_{A} P \sin (\pi \Theta) d A\right]^{2}+\left[\int_{A} P \cos (\pi \Theta) d A\right]^{2}
$$

From which the attitude angle, $\phi$, can also be computed as:

$$
\phi=\arctan \left(\frac{F_{X}}{F_{Y}}\right)
$$


The zero- and first-order terms of the dimensionless force components can then be obtained by expanding the square brackets and considering $d A=R d \theta d Z=\pi R L d \Theta d z=\pi R L d a, P=P_{p} p$ (neglecting $P_{E X T}$ ) and $p=p_{0}+\varepsilon p_{1}$. Then, for example, for the static condition is:

$$
\begin{aligned}
& f_{\Theta}{ }^{2}=\left(\frac{F_{X}}{\pi R L P_{p}}\right)^{2}=\left(\int_{A} p_{s 0} \sin (\pi \Theta) d a\right)^{2}+2 \varepsilon \int_{A} p_{s 1} \sin (\pi \Theta) d a \int_{A} p_{s 0} \sin (\pi \Theta) d a \\
& f_{y}{ }^{2}=\left(\frac{F_{Y}}{\pi R L P_{p}}\right)^{2}=\left(\int_{A} p_{s 0} \cos (\pi \Theta) d a\right)^{2}+2 \varepsilon \int_{A} p_{s 1} \cos (\pi \Theta) d a \int_{A} p_{s 0} \cos (\pi \Theta) d a
\end{aligned}
$$

Accordingly, the components of the static carrying capacity and the dynamic coefficients can be calculated from:

$$
\left.\begin{array}{l}
f_{i}=\sqrt{\left(f_{i 0}\right)^{2}+2 \varepsilon f_{i 0} f_{i 1}} \\
k_{i j}=\sqrt{\left(k_{i j 0}\right)^{2}+2 \varepsilon k_{i j 0} k_{i j 1}} \\
c_{i j}=\sqrt{\left(c_{i j 0}\right)^{2}+2 \varepsilon c_{i j 0} c_{i j 1}}
\end{array}\right\} i, j=y, \Theta
$$

where

$$
\begin{aligned}
& f_{\Theta k}=\int_{A} p_{s k} \sin (\pi \Theta) d a \quad ; \quad f_{y k}=\int_{A} p_{s k} \cos (\pi \Theta) d a \quad \text { with } k=0 ; 1 \\
& \left.\begin{array}{rl}
k_{\Theta \Theta k} & =\int_{A} p_{\Theta k} \sin (\pi \Theta) d a ; \quad k_{y y k}=\int_{A} p_{y k} \cos (\pi \Theta) d a \\
k_{y *} & =\int_{A} p_{\Theta A} \cos (\pi \Theta) d a \quad ; \quad k
\end{array}\right] \quad \text { with } k=0 ; 1 \\
& c_{\Theta \Theta k}=\int_{A} p_{\Theta k} \sin (\pi \Theta) d a ; \quad c_{y y k}=\int_{A} p_{y k} \cos (\pi \Theta) d a \\
& c_{y \Theta k}=\int_{A} p_{\Theta k} \cos (\pi \Theta) d a ; \quad c_{\Theta y k}=\int_{A} p_{j k} \sin (\pi \Theta) d a
\end{aligned}
$$

The procedure used to calculate the components of the force and, consequently, the dynamic coefficients gives only positive values (see Eq. (26)). While this would seem to be a disadvantage of the method, it does not incur in a big problem because all the coefficients calculated by finite differences also maintain their sign for all values of eccentricity and aspect ratio.

The parameters and coefficients that correspond to the ISJB are obtained considering $\varepsilon=0$ ( $k=0$ in Eqs. (27) to (29)) and integrating the equations in the $z$-direction. In this way, the following analytical results are obtained, 


$$
\begin{aligned}
& f_{y 0}=\frac{O_{0} \eta^{2}}{\pi\left(1-\eta^{2}\right)^{2}} ; \quad f_{\Theta 0}=-\frac{1}{4} \frac{O_{0} \eta}{\left(1-\eta^{2}\right)^{3 / 2}} \\
& O_{0}=\frac{8\left(1-\eta^{2}\right)^{2}}{\eta \sqrt{16 \eta^{2}+\pi^{2}\left(1-\eta^{2}\right)}} \\
& k_{\Theta \Theta 0}=\frac{O_{0} \eta}{\pi\left(1-\eta^{2}\right)^{2}} ; \quad k_{y y 0}=\frac{2 O_{0} \eta\left(\eta^{2}+1\right)}{\pi\left(1-\eta^{2}\right)^{3}} \\
& k_{\Theta y 0}=\frac{1}{4} \frac{O_{0} \eta}{\left(1-\eta^{2}\right)^{3 / 2}} \quad ; \quad k_{y \Theta 0}=-\frac{1}{4} \frac{O_{0}\left(2 \eta^{2}+1\right)}{\left(1-\eta^{2}\right)^{5 / 2}} \\
& c_{\Theta \Theta 0}=-\frac{1}{2} \frac{O_{0}}{\left(1-\eta^{2}\right)^{3 / 2}} \quad ; \quad c_{y y 0}=\frac{1}{2} \frac{O_{0}\left(2 \eta^{2}+1\right)}{\left(1-\eta^{2}\right)^{5 / 2}} ; \quad c_{\Theta y 0}=c_{y \Theta 0}=-\frac{2 O_{0} \eta}{\pi\left(1-\eta^{2}\right)^{2}}
\end{aligned}
$$

Similarly, the first-order terms of these parameters and coefficients ( $k=1$ in Eqs. (27) to (29)), are:

$$
\begin{aligned}
& f_{\Theta 1}=-\frac{1}{40} \frac{\left(11 O_{0}+10 O_{1}\right) \eta^{3}+\left(4 O_{0}-10 O_{1}\right) \eta}{\left(1-\eta^{2}\right)^{5 / 2}} \\
& f_{y 1}=\frac{1}{5} \frac{\left(6 O_{0}+5 O_{1}\right) \eta^{4}+\left(6 O_{0}-5 O_{1}\right) \eta^{2}}{\pi\left(1-\eta^{2}\right)^{3}} \\
& O_{1}=-\frac{1}{10} \frac{O_{0}\left(\left(11 \pi^{2}-192\right) \eta^{4}+\left(-192-7 \pi^{2}\right) \eta^{2}-4 \pi^{2}\right)}{\left(\pi^{2}-16\right) \eta^{4}+\left(16-2 \pi^{2}\right) \eta^{2}+\pi^{2}} \\
& \left.k_{\Theta \Theta 1}=\frac{1}{5} \frac{\left(6 O_{0}+5 O_{1}\right) \eta^{2}+6 O_{0}-5 O_{\lambda}}{\pi\left(1-\eta^{2}\right)^{3}}\right) \\
& k_{y y 1}=\frac{2}{5} \frac{\left(\left(6 O_{0}+51 O_{1}\right) \eta^{4}+24 O_{0} \eta^{2}+6 O_{0}-5 O_{1}\right) \eta}{\pi\left(1-\eta^{2}\right)^{4}} \\
& k_{\Theta y 1}=\frac{1}{40} \frac{\left(11 O_{0}+10 O_{\ell}\right) \eta^{2}+4 O_{0}-10 O_{1}}{\left(1-\eta^{2}\right)^{5 / 2}} \\
& k_{y \Theta 1}=\frac{1}{40} \frac{\left(22 O_{0}+20 O_{1}\right) \eta^{4}+\left(49 O_{0}-10 O_{1}\right) \eta^{2}+4 O_{0}-10 O_{1}}{\pi\left(1-\eta^{2}\right)^{7 / 2}}
\end{aligned}
$$




$$
\begin{aligned}
& c_{\Theta \Theta 1}=\frac{1}{20} \frac{\left(11 O_{0}+10 O_{1}\right) \eta^{2}+4 O_{0}-10 O_{1}}{\left(1-\eta^{2}\right)^{5 / 2}} \\
& c_{y y 1}=\frac{1}{20} \frac{\left(\left(16 O_{0}+20 O_{1}\right) \eta^{4}+\left(25 O_{0}-10 O_{1}\right) \eta^{2}+4 O_{0}-10 O_{1}\right)}{\left(1-\eta^{2}\right)^{7 / 2}} \\
& c_{\Theta y 1}=-\frac{2}{5} \frac{\left(\left(6 O_{0}+5 O_{1}\right) \eta^{2}+6 O_{0}-5 O_{1}\right) \eta}{\pi\left(1-\eta^{2}\right)^{3}} \\
& c_{y \Theta 1}=-\frac{2}{5} \frac{\left(\left(4 O_{0}+5 O_{1}\right) \eta^{2}-5 O_{1}\right) \eta}{\pi\left(1-\eta^{2}\right)^{3}}
\end{aligned}
$$

\section{RESULTS}

In this section, the results obtained using the proposed perturbation method are compared to those from the numerical solution of the Reynolds equation and the Ocvirk solution. The three sets of results are identified, respectively, as "P\&O" (referencing the fact that both, pressure and Ocvirk number are expanded), "Num" and "ISJB". The comparison is carried out in the whole range of eccentricity ratios $(0<\eta<1)$ for $L / D=0.25,0.5,0.75$ and 1.0. Thus, the perturbation parameter, $\varepsilon=(L / 2 D)^{2}$, takes the values $0.015625,0.0625,0.140625$ and 0.25 , respectively. The new expression of the perturbation parameter gives place to smaller corrections over the ISJB solution than those obtained in the previous work (with $\varepsilon=(L / D)^{2}$ ), for a given aspect ratio, improving the predictions of most static parameters and of the dynamic coefficients. The only coefficient that somehow resents the change introduced in the expression of $\varepsilon$ is the friction coefficient, but, as it will be seen further down, the predictions are still an improvement over the ISJB.

\subsection{Static Results}

In the previous work, it was already demonstrated that the pressure profiles, as well as the Ocvirk number and the dimensionless friction coefficient, are better described by the $\mathrm{P} \& \mathrm{O}$ method than by equivalent methods in which just the pressure is expanded (either by considering $p_{1}$ just a function of $O_{0}$, or by calculating the Ocvirk number with the expanded expression of the pressure). The results corresponding to the three mentioned parameters are again briefly discussed in this Section, given the fact that a new expression of the perturbation parameter is used. The phase angle and flow rate, which were not presented in the first work, 
are now included.

\section{Ocvirk number}

Figure 4 shows the Ocvirk number as a function of eccentricity ratio for the four chosen values of $L / D$. The numerical results as well as the predictions of the ISJB solution $\left(O_{0}\right)$ and the $\mathrm{P} \& \mathrm{O}$ approximation $\left(O=O_{0}+\varepsilon O_{1}\right)$, according to Eqs. (30) and (31), are shown. The distance between the curves of the ISJB approximation and the $\mathrm{P} \& \mathrm{O}$ corresponds to the firstorder correction introduced by the proposed method. As expected, all results coincide at small
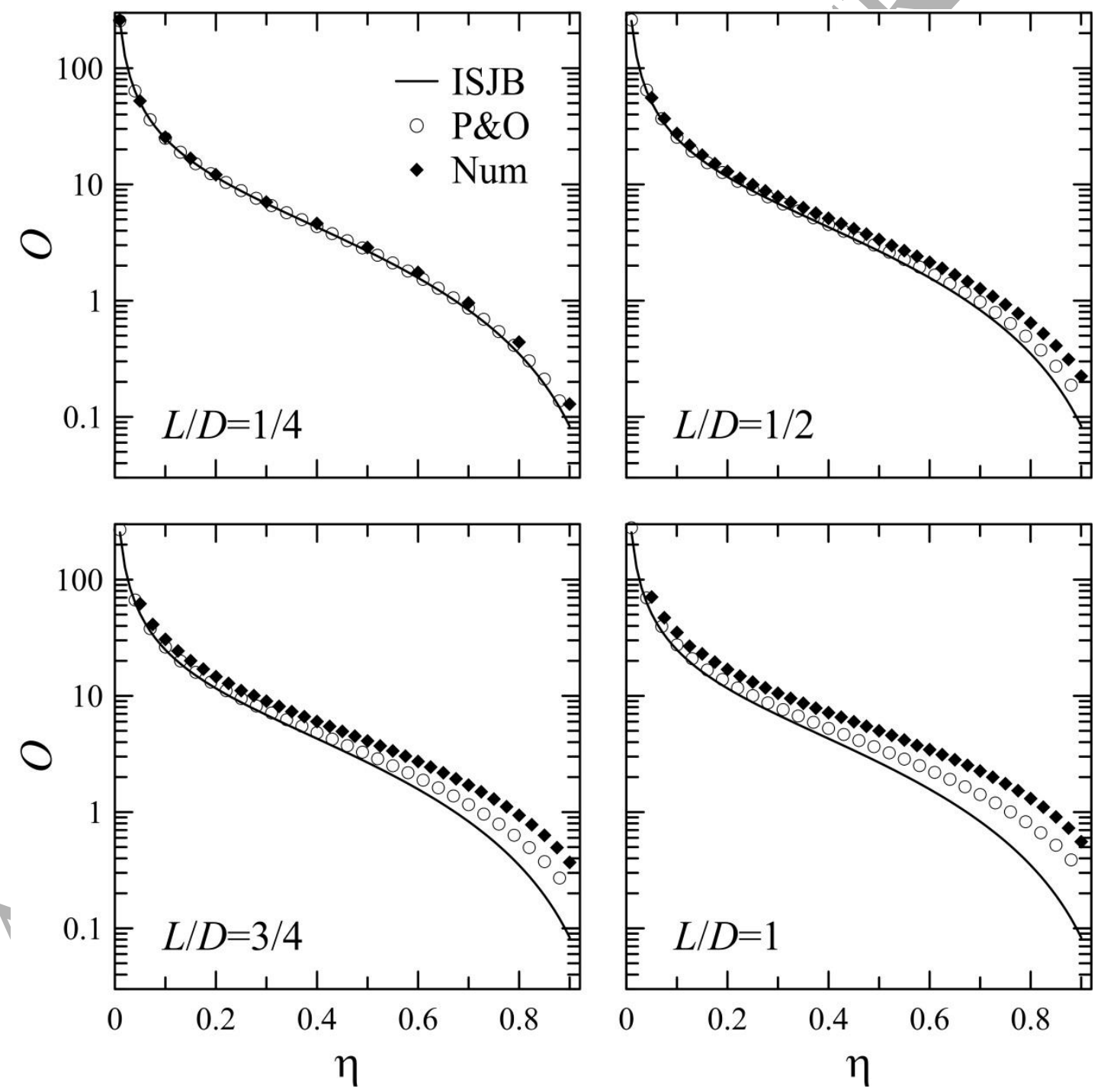

Figure 4. Ocvirk number as a function of eccentricity ratio for different aspect ratios.

Exact (Num) and approximate (P\&O) solutions compared to the Ocvirk solution for infinitely short JB (ISJB). 
eccentricity ratios (for all $L / D$ ) and small aspect ratios (at all eccentricities). As the aspect ratio increases, the Ocvirk number at a given eccentricity increases (see the Num curves), deviating from the ISJB solution. The $\mathrm{P} \& \mathrm{O}$ method also gives Ocvirk numbers that increase with $L / D$, but the predicted increment is not as large as it is shown by the numerical results. So, for example, in the limit case of $L / D=1$ and $\eta=0.8$, where the value of $O$ is underestimated by the ISJB approximation by a factor of about 3.5, the P\&O gives a value that is less than two times smaller than the exact value. At these same conditions, the use of $\varepsilon=(L / D)^{2}$ overestimates the value of $O$ by more than $50 \%$ [4].

\section{Pressure profiles}

Figures 5 and 6 present some representative dimensionless pressure/profiles. In the first one, the pressure is displayed as a function of the azimuthal direction $(\Theta)$ calculated at $z=0$ for $\eta=0.5$ and $L / D=0.75$. On the other hand, Figure 6 shows the dimensionless pressure as a function of aspect ratio (for $\eta=0.5$ ) and eccentricity ratio (for $L / D=0.5$ ) at the position $\Theta=0.5$ and $\mathrm{z}=0$. All results were obtained considering the Gümbel's (or $\pi$ ) boundary condition [2]. The pressure profiles change noticeably with position and conditions, but those presented in the figures are representative of the relative value and shape of the curves corresponding to the numerical results and the predictions. As it may be appreciated, the predictions of the $\mathrm{P} \& \mathrm{O}$ are close to the numerical solution and they represent an improvement with respect to the ISJB, as already proven in Vignolo et al. [4]. For example, for the chosen conditions of Figure 5, the azimuthal position of the maximum pressure practically matches that of the numerical results, with a value that is overestimated just by $2.6 \%$. This method predicts a pressure field that practically matches the exact solution up to $L / D=0.75$ and up to $\eta=0.75$ (as shown in Figure 6 for $\eta=0.5$ and $L / D=0.5$, respectively). In general, the results of dimensionless pressure profiles demonstrate that the $\mathrm{P} \& \mathrm{O}$ method captures very well the physics of the flow, at least up to aspect ratios of order one. 


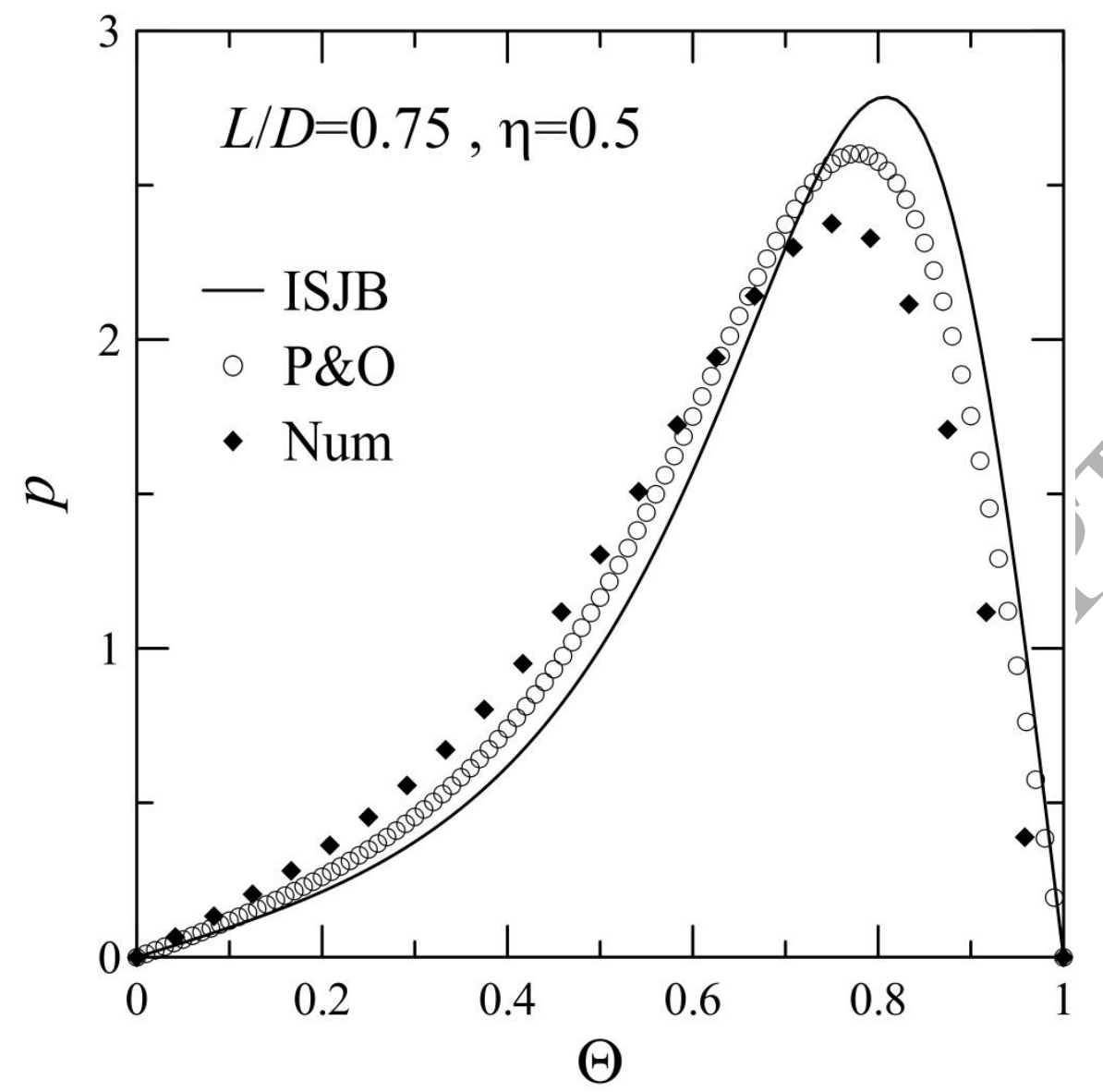

Figure 5. Dimensionless pressure profiles predicted at $z=0$ for $L / D=0.75$ and $\eta=0.5$.

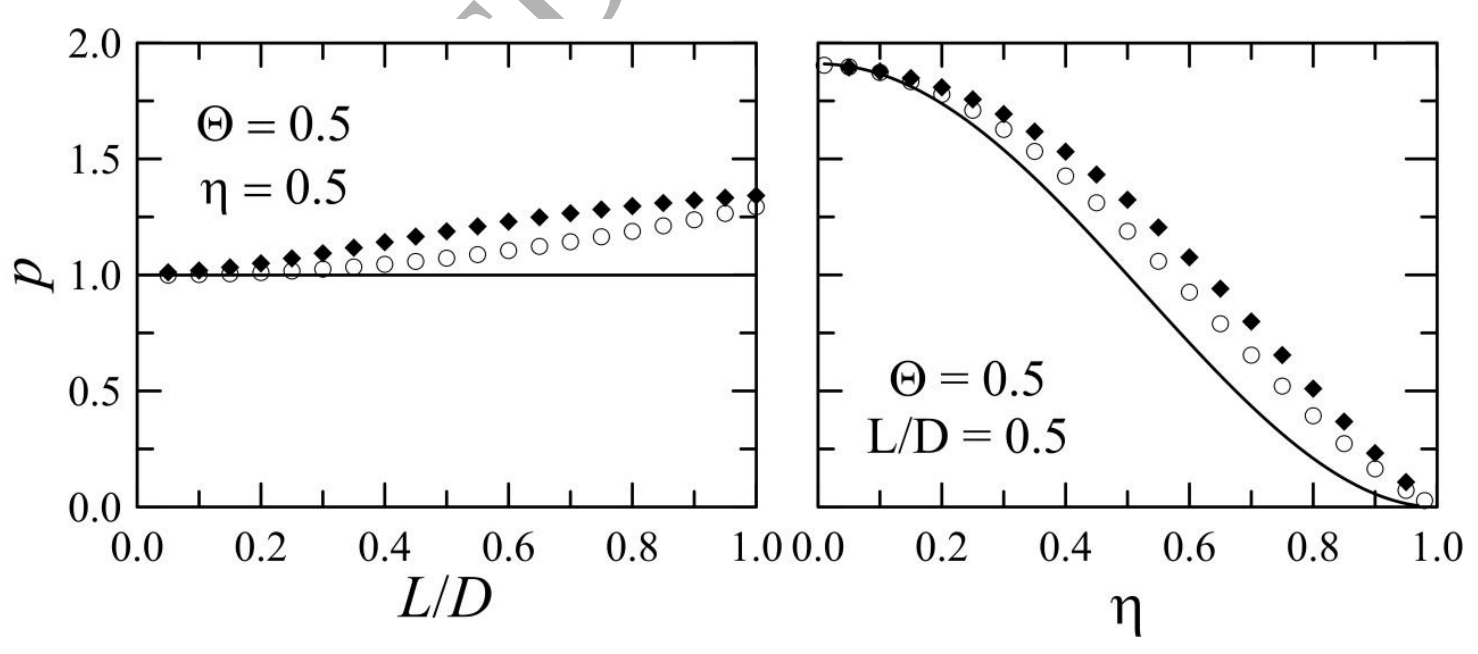

Figure 6. Dimensionless pressure profiles at $z=0$ and $\Theta=0.5$ as a function of aspect ratio (left, $\eta=0.5$ ) and eccentricity (right, $L / D=0.5$ ). 


\section{Friction coefficient}

The shear stress can be associated to the loss of power by friction in the JB. So, its integration over the journal area allows calculating the friction force, $F_{f}$, [4]

$$
F_{f}=\left.\int_{0}^{2} \int_{-1 / 2}^{1 / 2} \tau\right|_{h} \pi R L d \Theta d z=f_{f} F
$$

where the shear stress at the moving wall, or its dimensionless equivalent, $T_{w}$, can be calculated from Eq. (4) considering a non-slip boundary condition, as:

$$
T_{w}=-\left.\frac{\partial u}{\partial y}\right|_{h}=\frac{\left.\tau\right|_{h} c_{R}}{\mu U}=-\left(\frac{1}{\pi O}\left(\frac{L}{R}\right)^{2} \frac{\partial p_{s}}{\partial \Theta} \frac{h}{2}+\frac{1}{h}\right)
$$

As stated in Eq. (32), the friction force defines a friction coefficient, $f_{f}$, when divided by the load carrying capacity. The dimensionless friction coefficient is then obtained combining the order of magnitude of $F_{f}$ and $F$ (see Eq. (20)), as $f_{f} \frac{R}{c}\left(\frac{L}{R}\right)^{2}$.

Furthermore, the $\pi$-film hypothesis allows to assume a linear velocity profile in the divergent zone of the fluid film $(1<\Theta<2)[4]$, which combined with the above equations, gives the following equation to calculate the dimensionless friction coefficient,

$$
f_{f} \frac{R}{c}\left(\frac{L}{R}\right)^{2}=4 \varepsilon \int_{0}^{1} \int_{-1 / 2}^{1 / 2} h \frac{\partial p_{s}}{\partial \Theta} d \Theta d z+\frac{1}{2} \pi O \int_{0}^{2} \int_{-1 / 2}^{1 / 2} \frac{1}{h} d \Theta d z
$$

This equation clearly shows that the zero-order friction coefficient (which is also the ISJB solution) is given by the second term of the equation (which corresponds to the Couette flow between the moving walls) in its zero-order expression. Two integral terms then introduce the first-order correction to that solution; the first term of Eq. (34) evaluated using the zero-order expression of the pressure and the second one weighted by $O_{1}$. The analytical expression of the dimensionless friction coefficient according to the $\mathrm{P} \& \mathrm{O}$ method is:

$$
\begin{array}{r}
f_{f} \frac{R}{c}\left(\frac{L}{R}\right)^{2}=\frac{8 \pi\left(1-\eta^{2}\right)^{3 / 2}}{\eta \sqrt{16 \eta^{2}+\pi^{2}-\pi^{2} \eta^{2}}}+4 \varepsilon \frac{\left(1-\eta^{2}\right)^{3 / 2}}{\sqrt{16 \eta^{2}+\pi^{2}-\pi^{2} \eta^{2}}}(2 \pi- \\
\left.-\frac{4}{5} \frac{\left(11 \pi^{2} \eta^{4}-192 \eta^{4}-192 \eta^{2}-7 \pi^{2} \eta^{2}-4 \pi^{2}\right)}{\eta\left(-16 \eta^{4}-\pi^{2} \eta^{4}+16 \eta^{2}-2 \pi^{2} \eta^{2}-\pi^{2}\right)}\right)
\end{array}
$$

where the expression for the ISJB is that in the first term. Figure 7 displays the dimensionless 
friction coefficient as a function of eccentricity for the four aspect ratios. The values predicted by the P\&O show a considerable improvement over the ISJB solution, even for aspect ratios and eccentricities near 1 . In fact, the accuracy of the P\&O in the calculation of the friction coefficient extends up to $L / D \sim 1$ and $\varepsilon \sim 0.9$.
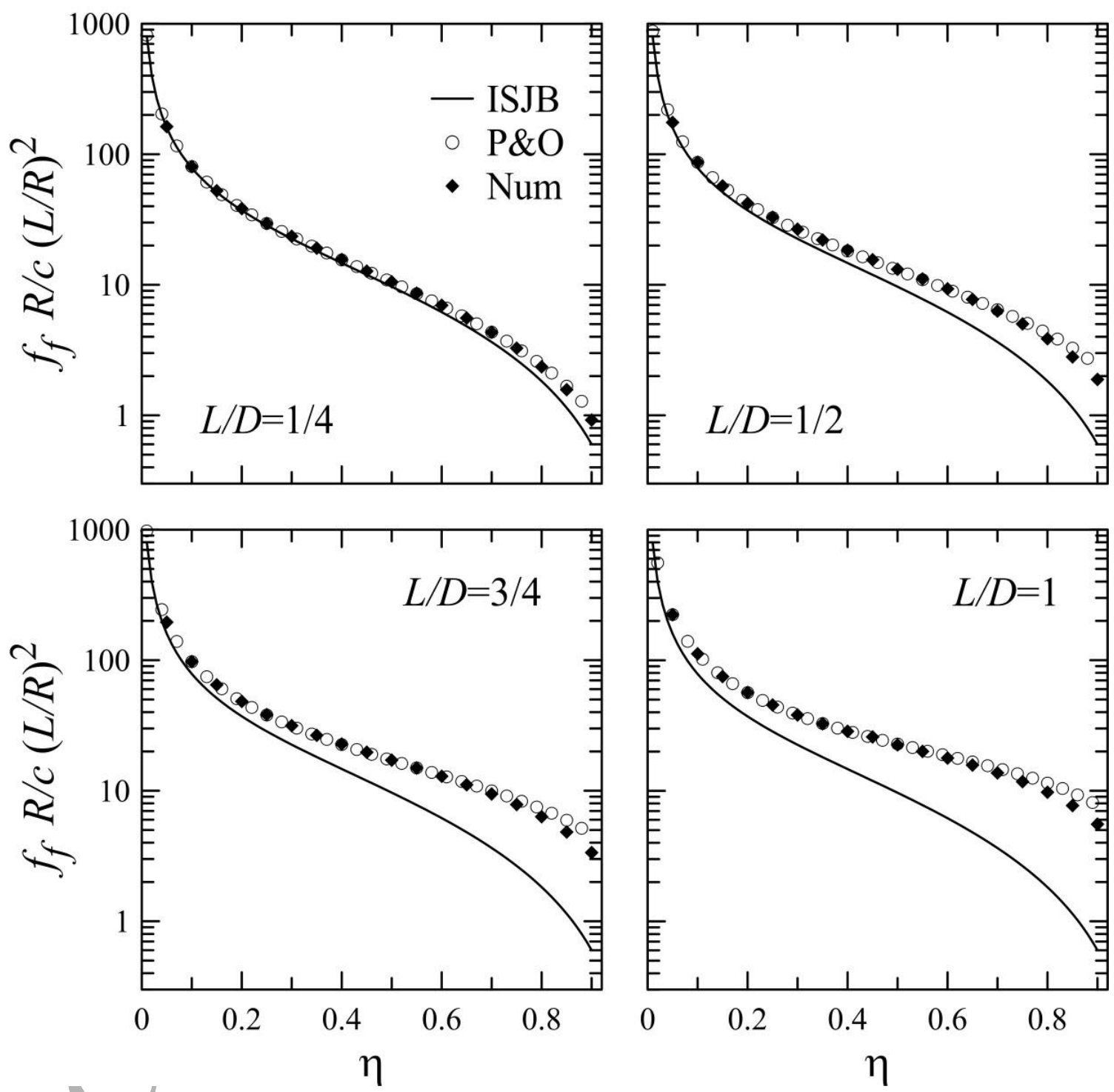

Figure 7. Dimensionless friction coefficient as a function of eccentricity for different aspect ratios.'

\section{Lubricant Flow rate}

The lubricant is constantly introduced into the JB at a rate that matches the fluid flow rate at which it exits through the edges (at $z= \pm 1 / 2$ ). Therefore, the flow rate of lubricant to be 
supplied to the bearing $\left(Q_{\mathrm{s}}\right)$ can be calculated by integrating the axial velocity of the fluid at the outlet sections. For example, at $z=1 / 2$, would be:

$Q_{s}=\left.\int_{0}^{2 \pi} \int_{0}^{H} V_{Z}\right|_{Z=L / 2} R d Y d \theta=\left.\frac{\pi U c_{R} R^{2}}{L} \int_{0}^{2} \int_{0}^{h} w\right|_{z=1 / 2} d y d \Theta$

where it has been taken into account the order of magnitude of $Y$ and $V_{\mathrm{Z}}$ listed in Eq. (7) and the fact that $W=\frac{U R}{L}$. The axial velocity profile, which can be obtained by integrating Eq.

(6) after changing the expression of $P_{\mathrm{REF}}$, is:

$$
w=\frac{1}{2 O}\left(\frac{L}{R}\right)^{2} \frac{\partial p_{s}}{\partial z}\left(y^{2}-h y\right)
$$

Replacing Eq. (37) in Eq. (36), and considering the Gümbell condition in the diverging section of the $\mathrm{JB}$, is:

$$
Q_{s}=-\left.\frac{\pi}{12} \frac{U c_{R} L}{O} \int_{0}^{1} h^{3} \frac{\partial p_{s}}{\partial z}\right|_{z=1 / 2} d \Theta
$$

The dimensionless flow rate can then be defined as:

$$
q_{s}=\frac{Q_{s}}{2 \pi R c_{R} W}=\frac{Q_{s}}{2 \pi c_{R} U L}=-\left.\frac{1}{24 O} \int_{0}^{1} h^{3} \frac{\partial p_{s}}{\partial z}\right|_{z=1 / 2} d \Theta
$$

To obtain the analytical expression of the flow rate predicted by the perturbation method, $p_{\text {s }}$ and $O$ in Eq. (39) must be replaced by their expanded expressions. Then, since the Ocvirk number appears in the denominator of this equation, the expression of order $\varepsilon$ of the flow rate is calculated by multiplying both numerator and denominator by $O_{0}-\varepsilon O_{1}$ (which leaves just terms of order zero and two in the denominator) and then neglecting all terms of order two. The resulting expression of the dimensionless flow rate is:

$$
q_{s}=-\frac{1}{24}\left[\left.\frac{1}{O_{0}} \int_{0}^{1} h^{3} \frac{\partial p_{s 0}}{\partial z}\right|_{z=1 / 2} d \Theta+\left.\varepsilon \frac{1}{O_{0}} \int_{0}^{1} h^{3} \frac{\partial p_{s 1}}{\partial z}\right|_{z=1 / 2} d \Theta-\left.\varepsilon \frac{O_{1}}{O_{0}^{2}} \int_{0}^{1} h^{3} \frac{\partial p_{s 0}}{\partial z}\right|_{z=1 / 2} d \Theta\right]
$$

which, when solved using the expressions of $p_{\mathrm{s} 0}$ and $p_{\mathrm{s} 1}$ in the Appendix $\mathrm{B}$, gives: 


$$
\begin{aligned}
q_{s}=\frac{\eta}{4 \pi}+\varepsilon & \left(\frac{\eta\left(43 \pi^{2} \eta^{6}-84 \pi^{2} \eta^{4}+39 \pi^{2} \eta^{2}+2 \pi^{2}-736 \eta^{6}+320 \eta^{4}+416 \eta^{2}\right)}{120 \pi\left(16 \eta^{2}+\pi^{2}-\pi^{2} \eta^{2}\right)\left(-1+\eta^{2}\right)^{2}}+\right. \\
& \left.+\frac{\eta\left(11 \pi^{2} \eta^{4}-192 \eta^{4}-192 \eta^{2}-7 \pi^{2} \eta^{2}-4 \pi^{2}\right)}{40 \pi\left(\pi^{2} \eta^{4}-2 \pi^{2} \eta^{2}-16 \eta^{4}+16 \eta^{2}+\pi^{2}\right)}\right)
\end{aligned}
$$

Elaborating further, Eq. (41) can actually be simplified to:

$$
q_{s}=\frac{\eta}{4 \pi}\left(1-\frac{\varepsilon}{3}\right)
$$

that includes the solution for the ISJB (when $\varepsilon=0$ ).

Figure 8 displays the predicted results as well as the numerically calculated flow rates for the four $L / D$. The dimensionless lateral fluid flow increases with the eccentricity ratio and slightly decreases with aspect ratio. This is due to the effect that these parameters have over the difference between the centerline pressure and the external one. All results agree for very short JBs, but both, the ISJB and the P\&O predictions, overestimate the lateral flow as the aspect ratio increases. Actually, the improvement that the $\mathrm{P} \& \mathrm{O}$ represents in the estimation of the lubricant flow rate is not really significant, and its predictions fall short when compared with the Numeric results. In the case of flow rate, then, the first-order correction introduced by the method it is not sufficient, or the chosen perturbation parameter is too small.

\section{Phase angle}

The phase or attitude angle, $\phi$, measures the misalignment between the line of journal and bearing loci and the direction of the applied load. It is calculated as the ratio of the longitudinal to the transversal components of the fluid force, as shown in Eq. (24). 

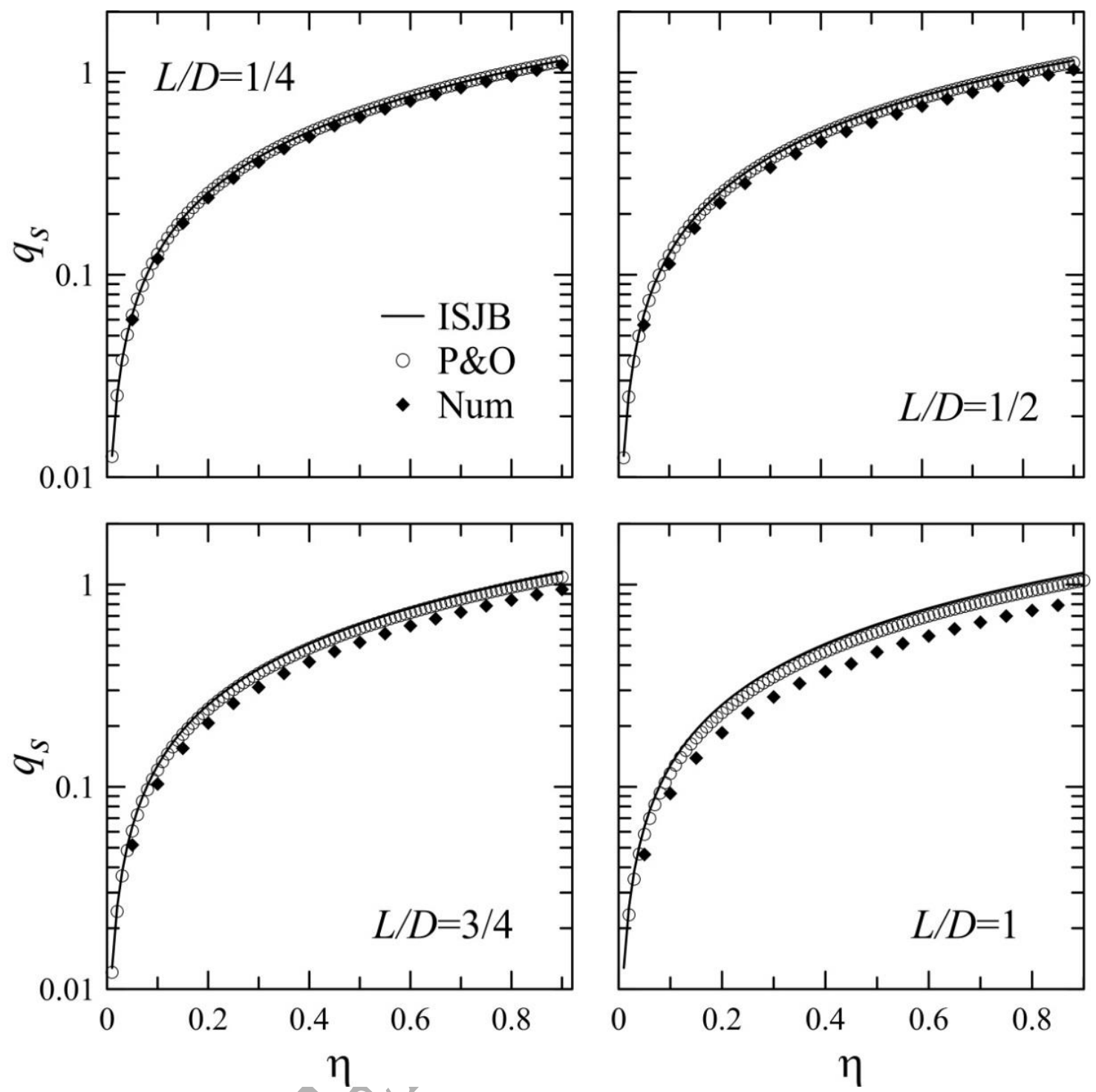

Figure 8. Dimensionless axial flow rate as a function of eccentricity for different aspect ratios.

The Ocvirk solution of the phase angle can then be obtained from Eq. (30). Its expression is:

$\phi_{0}=\arctan \left(\frac{\pi}{4} \frac{\sqrt{1-\eta^{2}}}{\eta}\right)$

The prediction of $\mathrm{P} \& \mathrm{O}$ arises from combining the expressions of the force components in Eqs. (30) and (31). Figure 9 displays the calculated numerical and analytical results of the dimensionless phase angle, $\psi=\phi / 2 \pi$. As observed, the ISJB solution underestimates the phase angle by an amount that increases with $\eta$ and $L / D$. The P\&O also underestimates $\psi$ at most conditions, but its predictions follow more closely the Numeric results, being practically 
identical at large eccentricity ratios. For instance, at $L / D=1$ and $\eta=0.8$, the ISJB solution underestimates the value of $\psi$ by $26 \%$ while the $\mathrm{P} \& \mathrm{O}$ does it just by $7 \%$.
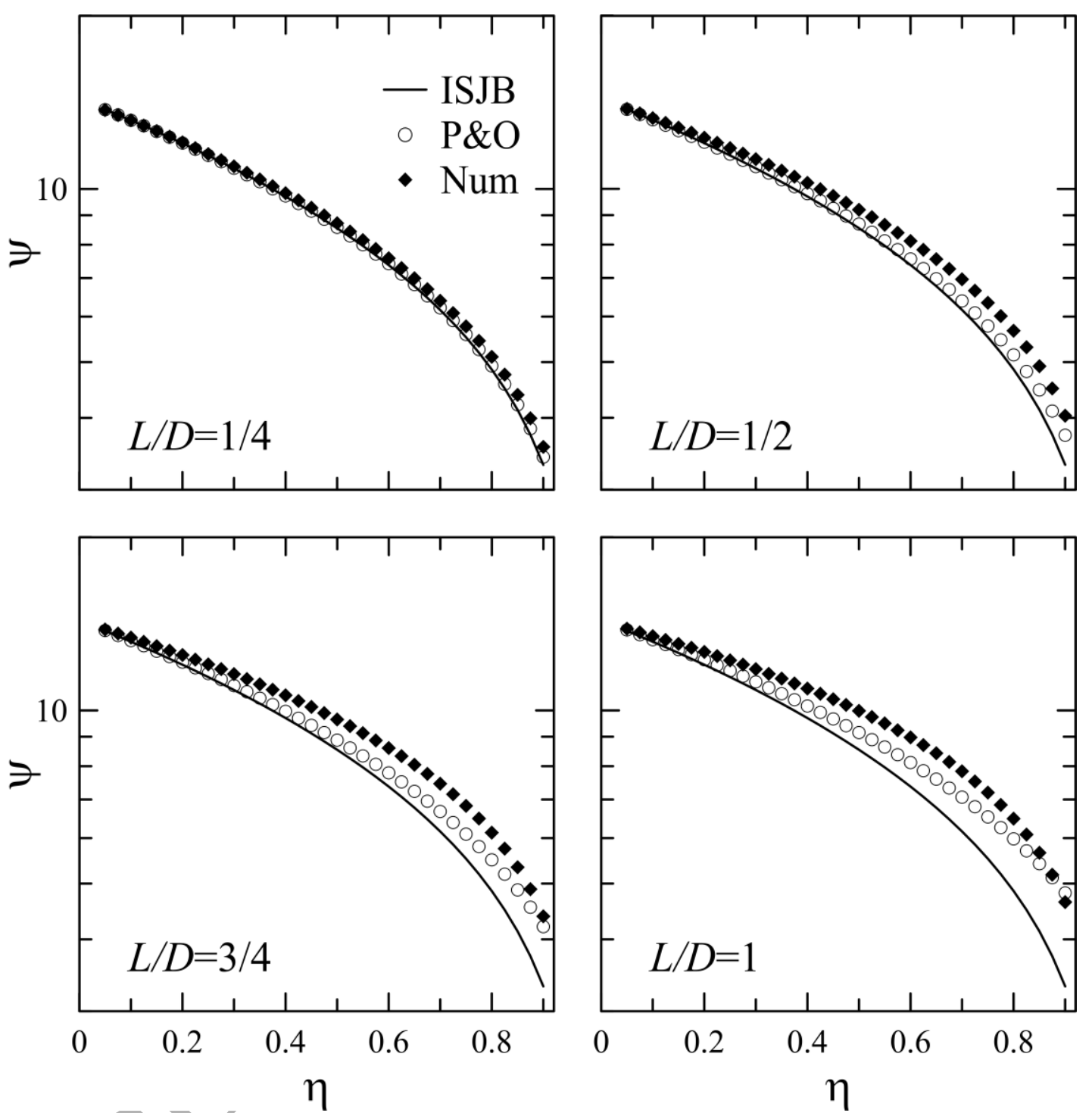

Figure 9. Dimensionless phase angle as a function of eccentricity for different aspect ratios.

\subsection{Dynamic Results}

In this Section, the dynamic coefficients predicted by the proposed method are compared to those from the Numeric and ISJB solutions. As before, the comparison is carried out for $0<\eta<1$ at the four chosen aspect ratios. The parameters are displayed referenced to the dimensionless static system of coordinates $\{\underline{x}, \underline{y}\}$ shown in Figure 2 instead of $\{\Theta, y\}$. The use of a static reference system allows for a deeper and more comprehensible analysis of the 
dynamic behavior of hydrodynamic bearings, and it facilitates the analyses of possible zones of instability. In fact, most studies in the literature represent the coefficients in this way [6$9,13,28,30,31]$. The transformation from the $\{\Theta, y\}$ coordinates to $\{\underline{x}, \underline{y}\}$ is done using the transformation matrix:

$\left(\begin{array}{ll}G_{\underline{x} \underline{x}} & G_{\underline{x y}} \\ G_{\underline{y} \underline{x}} & G_{\underline{y y}}\end{array}\right)=\left(\begin{array}{cc}\cos (\phi) & -\sin (\phi) \\ \sin (\phi) & \cos (\phi)\end{array}\right)\left(\begin{array}{ll}G_{\Theta \Theta} & G_{\Theta y} \\ G_{y \Theta} & G_{y y}\end{array}\right)\left(\begin{array}{cc}\cos (\phi) & \sin (\phi) \\ -\sin (\phi) & \cos (\phi)\end{array}\right)$

where $G$ stands for $k$ or $c$. The dynamic coefficients will be displayed in their dimensionless form (according to the order of magnitudes shown in Eq. (20)). By transforming the dynamic coefficients of the moving reference system to the static one, the cross-coupling stiffness coefficients, $k_{\underline{x y}}$ and $k_{\underline{\underline{x}} \underline{x}}$ may have opposite signs. In that case the coupling would give place to destabilizing forces that add energy to the orbit of the shaft, resulting in large amplitudes of displacement oscillation that cannot be cancelled by direct bearing dissipation [25].

\section{Stiffness coefficients}

Figures 10 and 11 display the dimensionless cross coupling and direct stiffness coefficients as a function of eccentricity for the four $L / D$. Actually, the cross-coupling stiffness coefficient $k_{\underline{v x}}$ is negative at small eccentricity ratios, and Figure 10 displays its absolute value. The change of sign occurs at $\eta=0.65$ for $L / D \rightarrow 0$ and at increasing values of $\eta$ as the aspect ratio augments (see the Numeric results). The change in sign of $k_{\underline{y x}}$ refers to the area of possible instability in the hydrodynamic bearings, which is associated to high Ocvirk numbers (small eccentricities) [32].

As can be seen in Figures 10 and 11, the predictions of the P\&O method match very well the values of $k_{y x}$ and $k_{y y}$ calculated numerically for a large range of eccentricity values and aspect ratios. Moreover, the eccentricity at which the change of sign of $k_{\underline{y x}}$ occurs is practically coincident, at least up to $L / D=3 / 4$, greatly improving the prediction of the ISJB solution. With respect to the direct stiffness coefficient $k_{\underline{x}}$, the correction introduced by the first-order term improves the ISJB solution at all $L / D$ and $\eta$, except at small eccentricities $(\eta<0.3)$ for high aspect ratios $(L / D>3 / 4)$. On the other hand, the cross-coupling stiffness coefficient $k_{\underline{x y}}$ is satisfactorily calculated by the proposed method except at eccentricities larger than $\sim 0.7$ for all aspect ratios, where the calculated values underestimate the coefficient even more than the ISJB. When analyzing $k_{\underline{x}}$, it has to be considered that this coefficient is 
the smallest of the four at small eccentricities (when the dimensional coefficients and the reaction force, which is in the denominator of the dimensionless expressions, have their smaller values). The logarithmic plot emphasizes, then, the differences that appear among the predictions as $L / D$ increases, even if they are not actually too large.
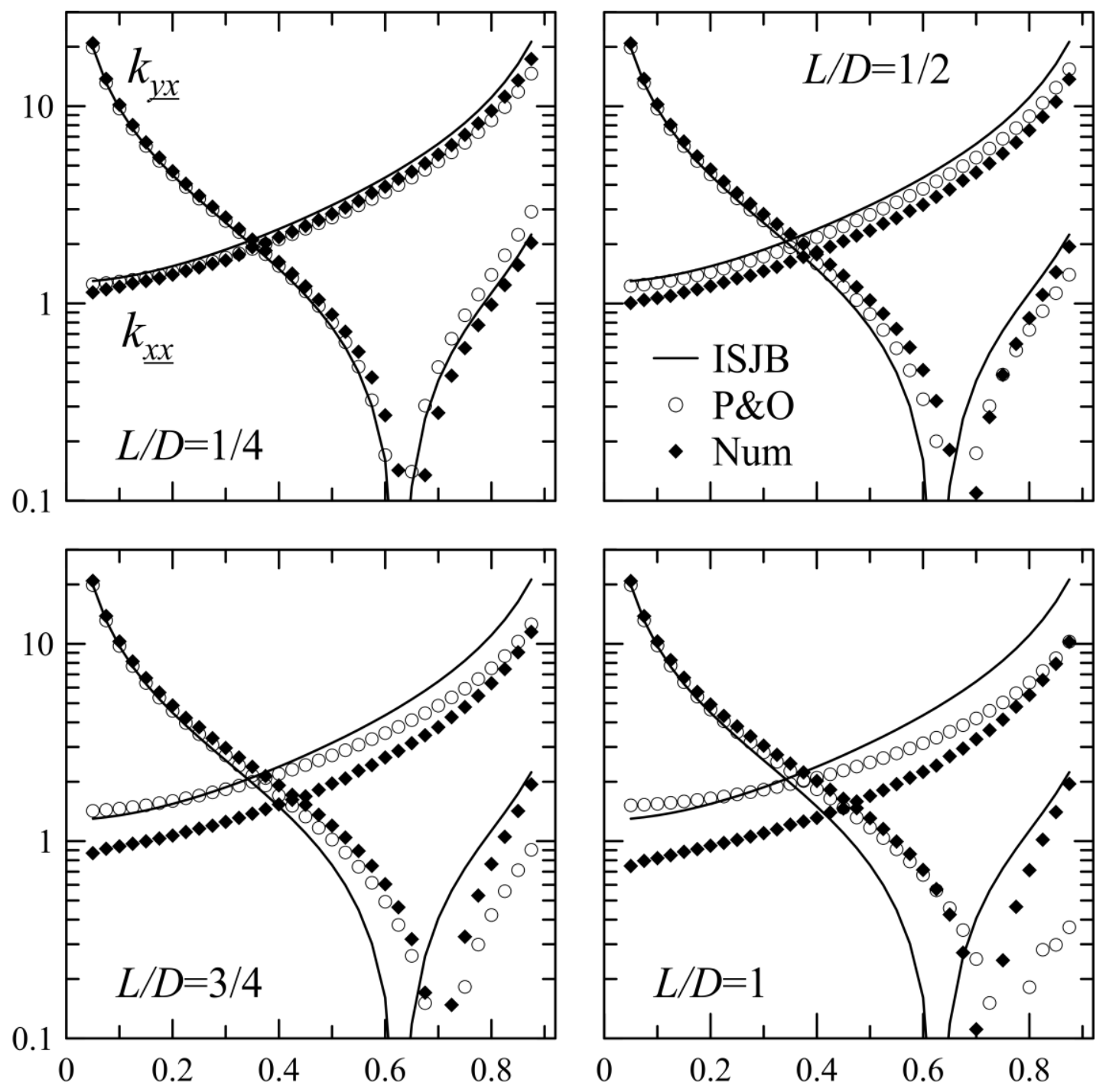

$\eta$

Figure 10. Dimensionless stiffness coefficients $k_{\underline{x x}}$ and $k_{\underline{y x}}$ as a function of eccentricity for different aspect ratios.

Summarizing, the regular perturbation method successfully extends the Ocvirk solution of the stiffness coefficients, giving excellent results in the whole range of eccentricities for aspect ratios smaller than about 3/4. Moreover, at $L / D>3 / 4$, the predicted results also represent 
an improvement over the estimations of the ISJB approximation at most eccentricities. The failure appears at large eccentricities in the case of $k_{\underline{y} \underline{ }}$ and $k_{\underline{y x}}$ ( $\eta$ larger than $\sim 0.6$ and $\sim 0.7$, respectively) and at small ones in case of $k_{\underline{x x}}$ ( $\eta$ smaller than $\left.\sim 0.3\right)$.
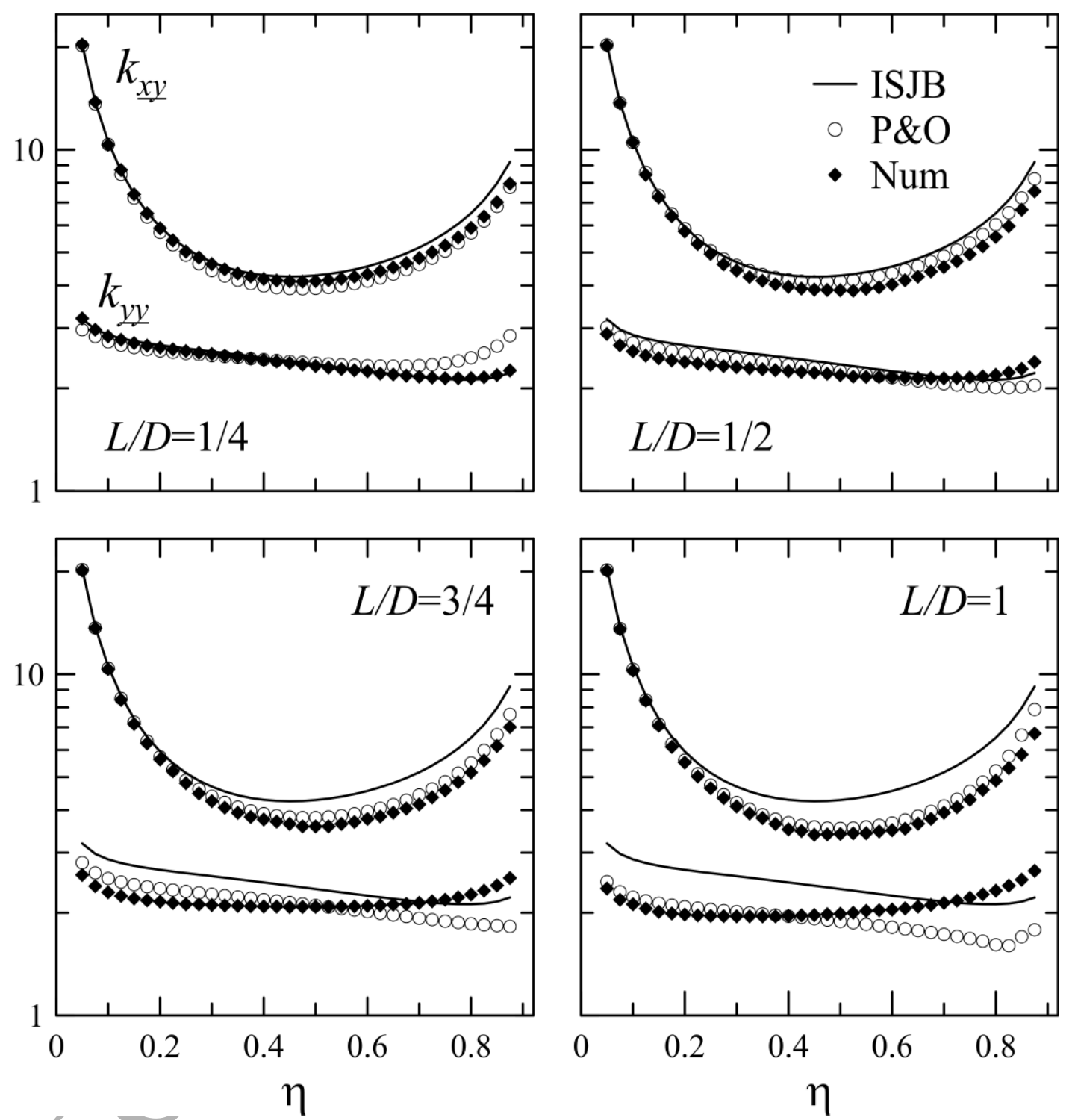

Figure 11. Dimensionless stiffness coefficients $k_{\underline{y} \underline{ }}$ and $k_{\underline{x y}}$ as a function of eccentricity for different aspect ratios.

\section{Damping coefficients}

During operation, hydrodynamic JBs present some advantages with respect to rolling bearings, like the ability to dissipate energy. That is why it is important to have mathematical tools which allow for the reliable prediction of the damping coefficients. In that sense, the 
cross-coupling damping coefficients characterize the stabilizing response of the system, while the direct ones are responsible of the dissipative capacity of the JB in case of impact and/or changes in the forces acting on the journal $[3,24]$.

Figure 12 and 13 present the dimensionless damping coefficients as a function of eccentricity for the four $L / D$ considered in this work. As expected, the predictions of the P\&O are very similar to those of the ISJB solution at $L / D=1 / 4$ for all eccentricities. These predictions agree with the numerical results at least up to $\eta$ of about 0.6. At larger eccentricities, they somehow underestimate at least three of the coefficients.
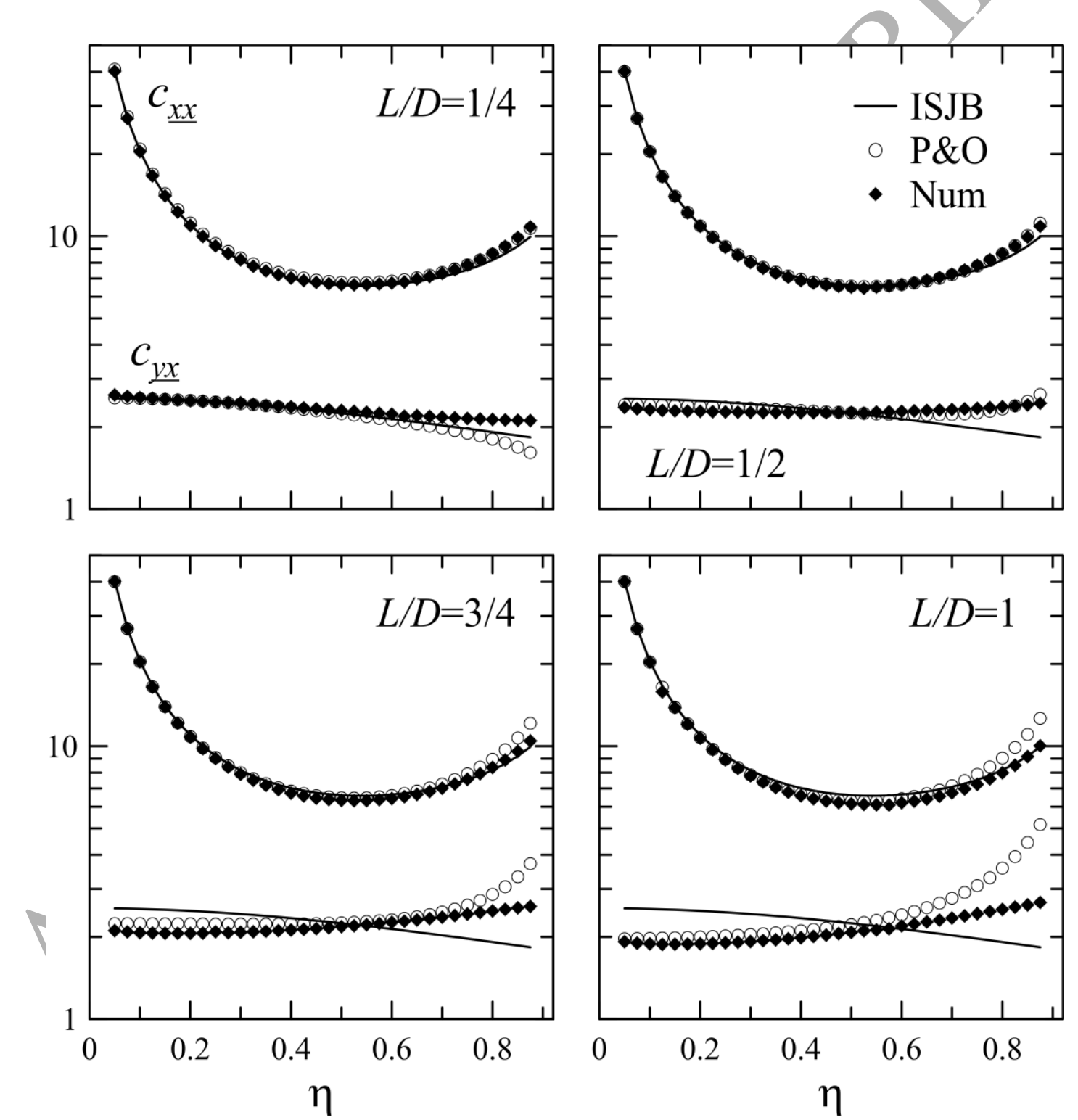

Figure 12. Dimensionless damping coefficients $c_{\underline{x x}}$ and $c_{\underline{y x}}$ as a function of eccentricity for different aspect ratios. 

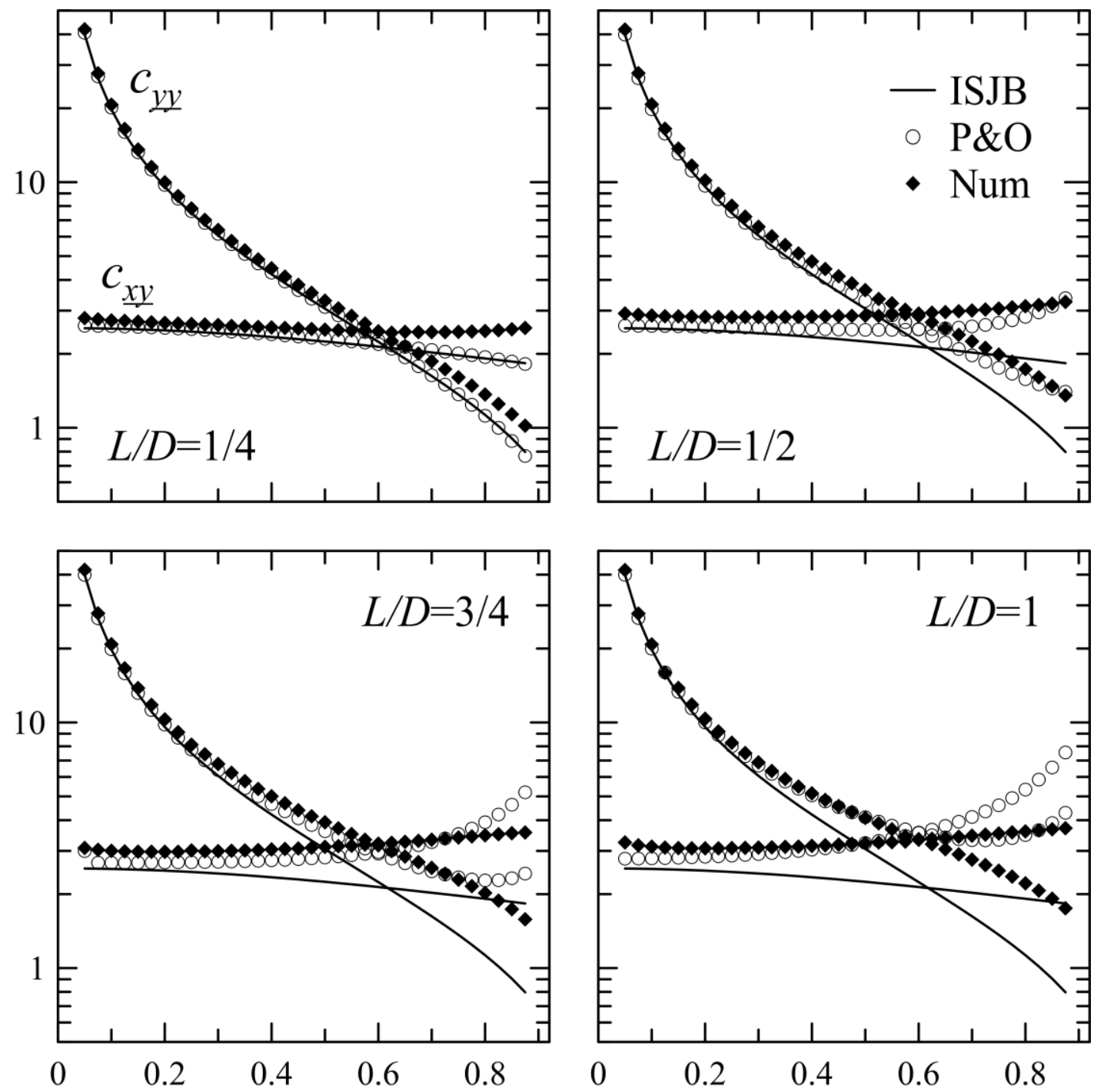

$\eta$

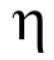

Figure 13. Dimensionless damping coefficients $c_{\underline{y y}}$ and $c_{\underline{x y}}$ as a function of eccentricity for different aspect ratios.

As $L / D$ increases, the numerical solution of the damping coefficients, except $c_{\underline{x x}}$, separate from the ISJB solution even at low eccentricities. It is interesting to observe that the firstorder correction introduced by the $\mathrm{P} \& \mathrm{O}$ method captures the nature of the dynamic phenomena taking place, producing predictions that closely follow the exact numerical results, even for $L / D=1$ at eccentricities smaller than $\sim 0.6$. In the case of $c_{\underline{x x}}$, there is practically no noticeable differences between the solutions as eccentricity and aspect ratio vary. It is important to observe that the difference between the cross-coupling damping coefficients, which it is not described by the ISJB solution but appears in published 
experimental data [19-22] and in numerical results, can actually be noticed in the proposed solution.

\section{Global error}

The improvement introduced by the $\mathrm{P} \& \mathrm{O}$ over the ISJB approximation in predicting the dynamic coefficients is clearly shown in Figure 14. This figure summarizes the global error of both methods by displaying the result of calculating:

$$
\sum_{\eta=0}^{0.9}\left|\log \left(\operatorname{coef}(\eta)_{\text {predicted }}\right)-\log \left(\operatorname{coef}(\eta)_{\text {Numeric }}\right)\right|
$$

where $\operatorname{coef}(\eta)$ stands for any of the eight dynamic coefficients at aiven $L / D$ and $\eta$. That is, the global error is calculated as the absolute value of the differences between the logarithmic values of a dynamic coefficient, summed over the thirty four eccentricity ratios considered in Figures 10 to 13 . Logarithms are considered so that all differences are of order of magnitude 1 .
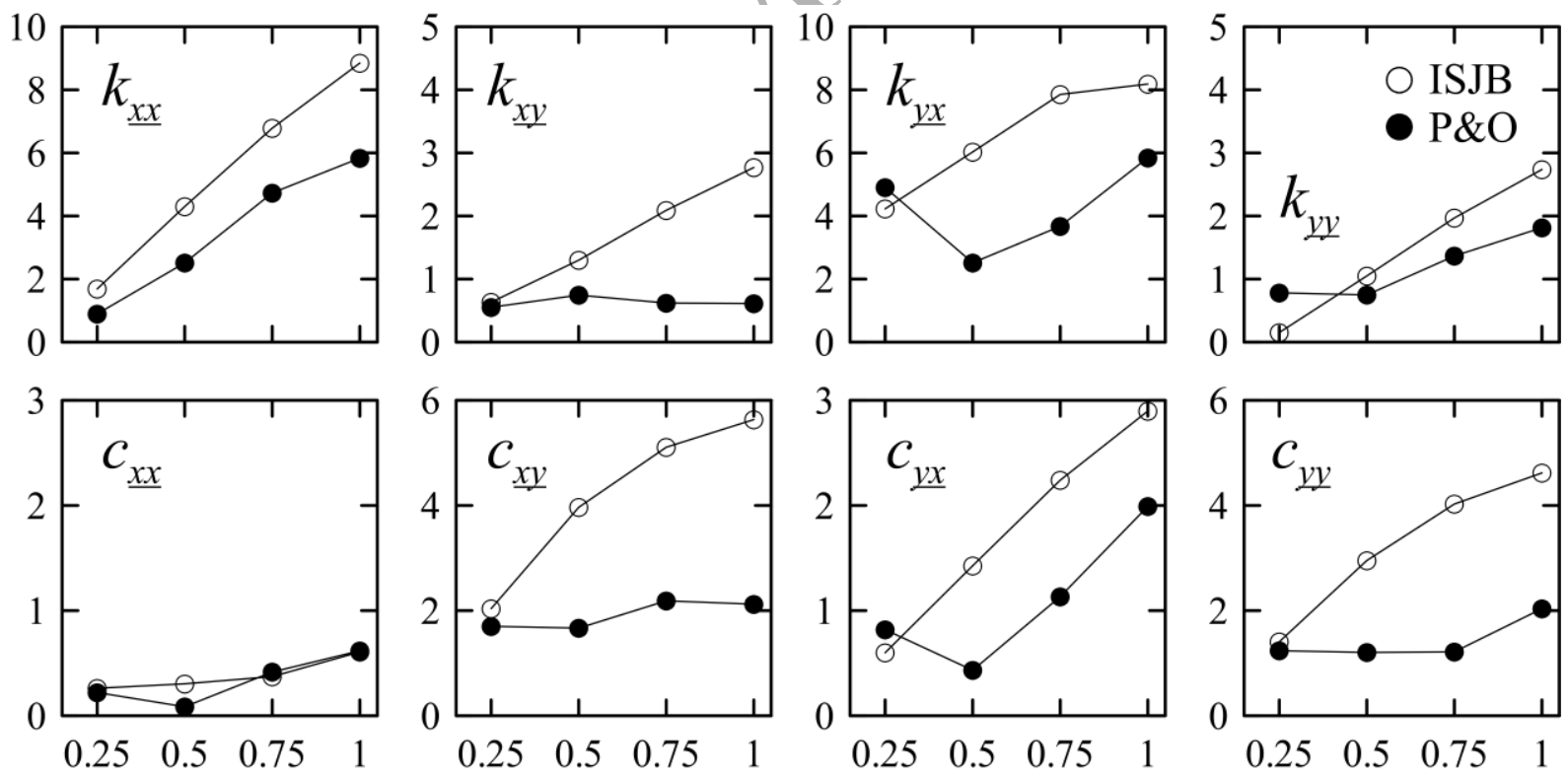

\section{$L / D$}

Figure 14. Global error of the ISJB approximation and the P\&O-perturbation in the calculation of the dynamic coefficients, calculated using Eq. (46). 
As expected, in all cases the global error of the ISJB increases as the aspect ratio augments from 0.25 to 1 . Apart from some cases at the smallest aspect ratio, the global error of the P\&O is always smaller, for all coefficients and all aspect ratios. Moreover, except in the case of $k_{\underline{y x}}$ and $c_{\underline{y x}}$, the rate of increase of the global error with $L / D$ is much smaller in the case of P\&O.

\section{Comparison with experimental results}

As commented in the Introduction Section, both, data and model predictions of the dynamic coefficients are sensitive to many design, processing, flow and fluid parameters and conditions, which difficult the comparison $[25,26]$. In this work we have chosen the data presented by Zhao et al. [21] for the comparison. To our best knowledge, this is the latest work that tests a plain-cylinder JB [26]. Besides, they use modern technology to reduce additional vibrations and the noise in the experimental data. These authors use a test rig with a rigid rotor and non-synchronous sinusoidal force input capability, that allow for static loads up to $40 \mathrm{kN}$, rotational frequencies up to $3600 \mathrm{rev} / \mathrm{min}$, and sinusoidal forces up to $1.5 \mathrm{kN}$. The bearing has a diameter of $152 \mathrm{~mm}$ with an aspect ratio $(L / D)$ of 0.581 and a radial clearance of $0.114 \mathrm{~mm}$. The lubricant considered is turbine oil 22 with a viscosity of $48.65 \times 10^{-3} \mathrm{~Pa}$.s at $20^{\circ} \mathrm{C}$ and $28.25 \times 10^{-3} \mathrm{~Pa}$.s at $30^{\circ} \mathrm{C}$. Excitation forces were measured using pressure sensors, displacements using eddy-eurrent probes, and velocities and accelerations were calculated using numerical differentiation with low-pass filtering, which is then used to reduce the additional vibrations generated by the other machine elements of the test rig.

Figure 15 displays the comparison between the dimensionless dynamic coefficients predicted by the $\mathrm{P} \& \mathrm{O}$ method and the experimental (Exp) and theoretical (THA) values informed by Zhao and coworkers, after been converted to the expressions of the variables presently used. Each Exp data shown in Figure 15 is centered in the quadratic polynomial fitting reported by the authors while the theoretical results correspond to the values calculated with a thermo-hydrodynamic analysis (coupled Reynolds and energy equations) using a finite element method. The exact solution of the Reynolds equation under isothermal condition (Num) is not included in Figure 16 since, at $L / D=0.58$, it would practically match the results of $\mathrm{P} \& \mathrm{O}$ (as it can be deduced from Figures 10 to 13 for $L / D=0.5$ and $\eta<0.6$, which corresponds to $\mathrm{O}>1$ ). The dispersion displayed by the experimental data are denoted in Figure 15 using grey ovals, which, given the logarithmic axis used, emphasize the dispersion when the load and/or coefficients are small. 

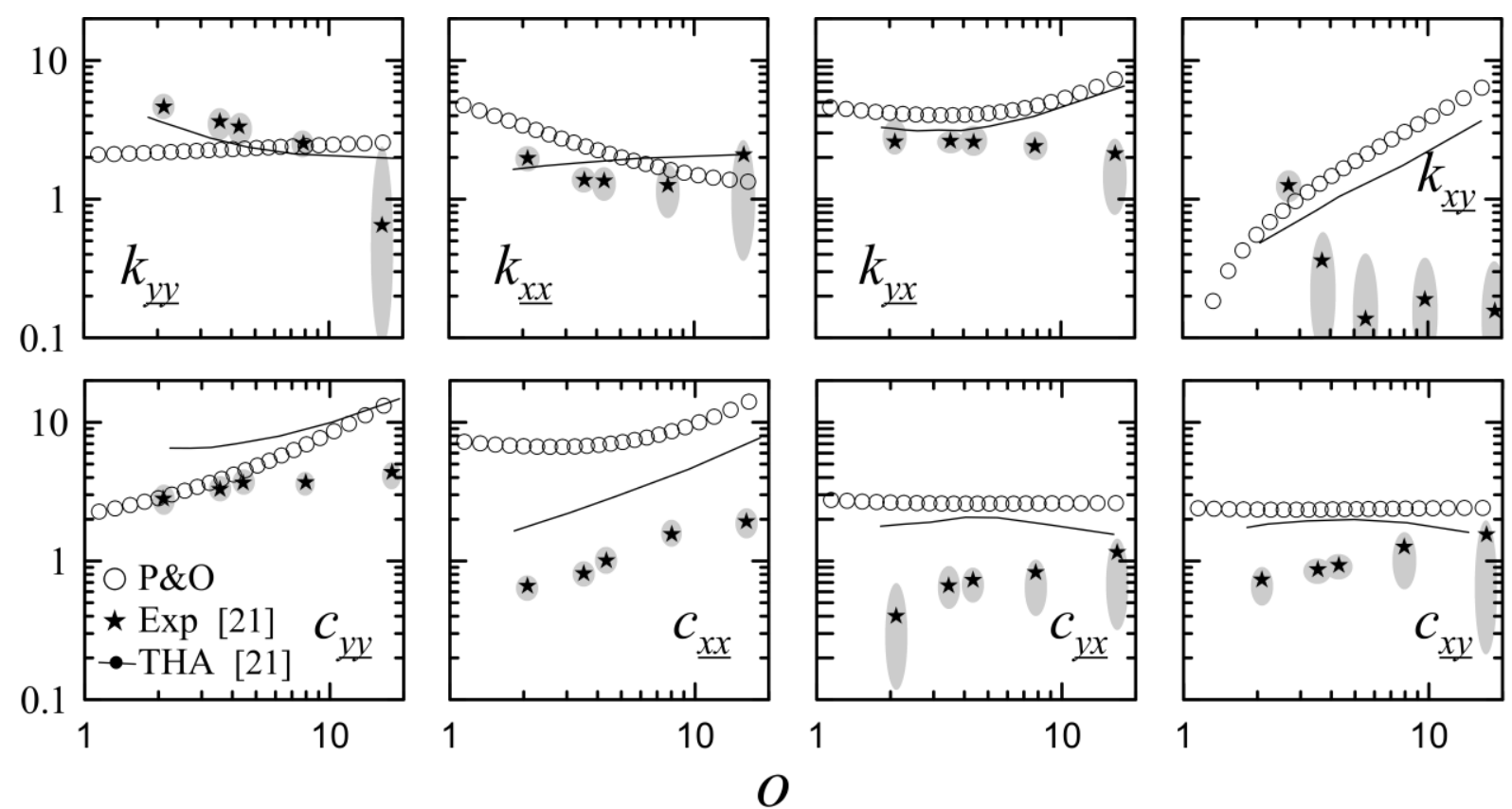

Figure 15. Dynamic coefficients (absolute values) as a function of Ocvirk number.

Comparison of experimental and theoretical data from Zhao et al. [21] for $L / D=0.58$ with predictions of the $\mathrm{P} \& \mathrm{O}$ model. The grey ovals indicate the dispersion in the experimental data.

The global observation of Figure 15 yields some important conclusions. First of all, the agreement between the predictions of the $\mathrm{P} \& \mathrm{O}$ model and the data is remarkably good, even more after considering that $\mathrm{P} \& \mathrm{O}$ is an isothermal model. Second, the qualitative behavior of the predicted coefficients as a function of $O$ is very similar to that of THA, showing in all cases the same trends. And third, not only most of the predicted values of the coefficients are not far from the theoretical ones of the THA but they are also close to the experimental data in many cases.

As it was already shown, low values of $\eta$ correspond to high values of $O$, and vice-versa (see Figure 4). Therefore, the results in Figures 10 and 11 indicate that the failure of $\mathrm{P} \& \mathrm{O}$ in predicting $k_{y \underline{y}}$ and $k_{\underline{x y}}$ should appear mainly at low values of $O$. Likewise, in the case of $k_{\underline{x x}}$, the failure should be at high values of $O$, while the cross-coupling stiffness coefficient $k_{\underline{y x}}$ should be satisfactorily calculated by the model in the whole range of Ocvirk number covered by the experimental study. The results in Figure 15, agree with these observations, mainly when the coefficients are compared with the THA results. When compared with the experimental data, 
a qualitative dissimilarity appears in the cross coupling coefficient $k_{\underline{x y}}$. In fact, the experimental data are positive at small values of $O$ and display a change in sign at an Ocvirk number about 7-8. This is not detected by neither model, which predict negative values of $k_{\underline{x y}}$ in the whole range of operational conditions considered. Even so, it should be observed that the $k_{x y}$ coefficient is the one that displays the smallest values, which justifies the apparently larger differences in the logarithmic scale.

In regard to the damping coefficients, and according the analysis presented in previous sections, the $\mathrm{P} \& \mathrm{O}$ model should give reasonable good predictions of all these coefficients, mainly at high values of $O$. The results in Figure 15 show that this is true. A very good agreement can be appreciated among the results of the cross damping coefficients in this situation. What is more, the predictions of $c_{\underline{x y}}$ and $c_{\underline{y x}}$ from the P\&O model are nearly coincident with those of the THA in all the operational range. $c_{y y}$ is overestimated at high values of $O$, but at low values the $\mathrm{P} \& \mathrm{O}$ model displays an excellent agreements with the measured coefficients, notably improving the result from the THA. $c_{\underline{x x}}$ is somehow overestimated in the whole range of loads by both models.

\section{Conclusions}

The comparison of the predictions from the proposed methodology with the exact results obtained by numerically solving the Reynolds equation confirm that the set of deduced expressions of load carrying capacity $(F)$, friction force $\left(F_{f}\right)$, attitude angle $(\phi)$, lubricant flow rate $\left(Q_{\mathrm{s}}\right)$ and stiffness $\left(K_{i j}\right)$ and damping $\left(C_{i j}\right)$ coefficients, as well as those of the pressure profiles and the Ocvirk number, $O$, constitute a powerful tool that can be used in the design and analysis of hydrodynamic JBs with aspect ratio up to 1 . In fact, the proposed methodology successfully extends the Ocvirk solution to describe finite length JBs with aspect and eccentricity ratios up to 3/4 (or combinations of larger eccentricities with smaller aspect ratios or vice-versa).

The proposed method complements, by means of simple direct analytical expressions, the one proposed by Chasalevris and Sfyris [9,15], which correctly describes the behavior of finite length JBs of large aspect ratio $(L / D>1)$. Together, both sets of analytical expressions cover the whole range of aspect ratios.

Although most existing experimental data of dynamic coefficients correspond to flow conditions or JB's shapes [19-26] different to the ones used in this work, the comparison with 
the data obtained by Zhao et al. for $L / D=0.58$ [21] reveals that the proposed methodology is not only very simple but, even assuming isothermal conditions, gives coefficients that are of the right order the magnitude and that correctly capture the nature of the phenomena.

\section{ACKNOWLEDGMENTS}

The authors would like to thank the National Research Council of Argentina (CONICET), Universidad Nacional del Sur (UNS), and Universidad Nacional de la Patagonia "San Juan Bosco" (UNPSJB) for the financial support.

\section{Nomenclature}

$C_{i j} \quad$ Damping coefficients

$c_{i j} \quad$ Dimensionless damping coefficients, $c_{i j}=\frac{U c_{R}}{\pi R^{2} L P_{p}} C_{i j}$

$c_{R} \quad$ Radial clearance

$D \quad$ Bearing diameter

e Eccentricity

$F_{X}, F_{Y} \quad$ Fluid film force $(F)$ components

$f_{\Theta}, f_{y} \quad$ Dimensionless fluid film force components, $f_{i}=\frac{F_{i}}{\pi R L P_{p}}$

$F_{f} \quad$ Friction force

$f_{f} \quad$ Friction coefficient, $f_{f}=\frac{F_{f}}{F}$

$H \quad$ Fluid film gap

$h$ Dimensionless fluid film gap, $h=\frac{H}{c_{R}}$

$h_{s} \quad$ Dimensionless static fluid film gap

$h_{d} \quad$ Dimensionless dynamic fluid film gap

$K_{i j} \quad$ Stiffness coefficients

$k_{i j} \quad$ Dimensionless stiffness coefficients, $k_{i j}=\frac{c_{R}}{\pi R L P_{p}} K_{i j}$ 
L Bearing length

$O \quad$ Ocvirk number, $O=\frac{\mu U}{R P_{p}}\left(\frac{R}{c_{R}}\right)^{2}\left(\frac{L}{R}\right)^{2}$

$P \quad$ Lubricant pressure

$p \quad$ Dimensionless lubricant pressure, $p=\frac{P-P_{E X T}}{P_{p}}$

$p^{\prime} \quad$ Dimensionless lubricant pressure, $p^{\prime}=\frac{P-P_{E X T}}{P_{R E F}}$

$P_{E X T} \quad$ External pressure

$P_{p} \quad$ Mean pressure, $P_{p}=\frac{F}{2 R L}$

$P_{R E F} \quad$ Reference pressure, $P_{R E F}=\frac{\mu U}{R}\left(\frac{R}{c_{R}}\right)^{2}\left(\frac{L}{R}\right)^{2}$

$Q_{s} \quad$ Lubricant flow rate

$q_{s} \quad$ Dimensionless lubricant flow rate, $q_{s}=\frac{Q_{s}}{2 \pi R c_{R} W}$

$R \quad$ Journal radius

$R_{B} \quad$ Bearing radius

$S \quad$ Sommerfeld number, $S=\frac{\mu U}{R P_{p}}\left(\frac{R}{c_{R}}\right)^{2}$

T Time

$t \quad$ Dimensionless time, $t=\frac{T}{T_{c}}$

$T_{c} \quad$ Characteristic time, $T_{c}=\frac{R}{U}$

$T_{w}$ Dimensionless shear stress at the moving wall, $T_{w}=-\left.\frac{\partial u}{\partial y}\right|_{h}$

$U, V, W \quad$ Characteristic values of velocity components

$u, v, w \quad$ Dimensionless velocity components in moving reference system

$V_{X}, V_{Y}, V_{Z}$ Velocity components in moving reference system

$W \quad$ Acting load 
$X, Y, Z \quad$ Moving reference system of coordinates

$\Theta, y, z \quad$ Dimensionless moving reference system of coordinates

$\underline{\mathrm{X}}, \underline{\mathrm{Y}}, \underline{\mathrm{Z}} \quad$ Static reference system of coordinates

$\underline{x}, \underline{y}, \underline{z} \quad$ Dimensionless static reference system of coordinates

$\varepsilon \quad$ Perturbation parameter, $\varepsilon=\left(\frac{L}{2 D}\right)^{2}$

$\phi \quad$ Attitude angle

$\eta \quad$ Eccentricity ratio, $\eta=\frac{e}{c_{R}}$

$\mu \quad$ Lubricant viscosity

$\omega \quad$ Journal angular velocity

$\psi \quad$ Dimensionless attitude angle, $\psi=\frac{\phi}{2 \pi}$

\section{References}

[1] Hamrock BJ, Schmid SR, Jacobson BO. Fundamentals of fluid film lubrication. 2nd ed. New York: Marcel Dekker, Inc.; 2004.

[2] Szeri, AZ. Fluid film lubrication, 2nd ed., Cambridge Univ. Press: New York; 2010.

[3] Childs D. Turbomachinery rotordynamics: Phenomena, modeling, and analysis, WileyInterscience; 1993.

[4] Vignolo GG, Barilá DO, Quinzani LM. Approximate analytical solution to Reynolds equation for finite length journal bearings. Tribol Int 2011;44:1089-1099.

[5] Li Q, Wang W, Weaver B, Wood H. Model-Based Interpolation-Iteration Method for Bearing Coefficients Identification of Operating Flexible Rotor-Bearing System. Int J Mech Sci 2017;131132:471-479.

[6] Rao TVVLN, Biswas S, Hirani H, Athre K. An analytical approach to evaluate dynamic coefficients and nonlinear transient analysis of a hydrodynamic journal bearing. Tribol Trans 2000;43:109-115.

[7] Ebrat O, Mourelatos ZP, Vlahopoulos N, Vaidyanathan K. Calculation of journal bearing dynamic characteristics including journal misalignment and bearing structural deformation. Tribol Trans 2004;47:94-102. 
[8] Meruane V, Pascual R. Identification of nonlinear dynamic coefficients in plain journal bearings. Tribol Int 2008;41:743-754.

[9] Chasalevris A, Sfyris D. Evaluation of the finite journal bearing characteristics, using the exact analytical solution of the Reynolds equation. Tribol Int 2013;57:216-234.

[10] Hei D, Lu Y, Zhang Y, Liu F, Zhou C, Müller N. Nonlinear dynamic behaviors of rod fastening rotor-hydrodynamic journal bearing system. Arch Appl Mech 2015;85:855-875.

[11] Synnegård E, Gustavsson R, Aidanpää JO. Influence of cross-coupling stiffness in tilting pad journal bearings for vertical machines. Int J Mech Sci 2016;111-112:43-54.

[12] Smolík L, Hajžman M, Byrtus M. Investigation of bearing clearance effects in dynamics of turbochargers. Int J Mech Sci 2017;127:62-72.

[13] Maharshi K, Mukhopadhyay T, Roy B, Roy L, Dey S. Stochastic dynamic behaviour of hydrodynamic journal bearings including the effect of surface roughness. Int $\mathrm{J}$ Mech Sci 2018;142-143:370-383.

[14] Yuan H, Di-Gong C. Effects of partial-grooving on the performance of spiral groove bearings: analysis using a perturbation method. Tribol Int 1996;29(4):281-290.

[15] Jang GH, Lee SH. Determination of the dynamic coefficients of the coupled journal and thrust bearings by the perturbation method. Tribol Int 2006;22:239-246

[16] Kim HW, Jang GH, Ha HJ. A generalized Reynolds equation and its perturbation equations for fluid dynamic bearings with curved surfaces. Tribol Int 2012;50:6-15

[17] Reason BR, Narang IP. Rapid design and performance evaluation of steady-state journal bearings - A technique amenable to rapid design and performance evaluation of steady-state journal bearings- A technique amenable to programmable hand calculators. ASLE Trans 1982;25(4):429444.

[18] Sfyris D, Chasalevris A. An exact analytical solution of the Reynolds equation for the finite journal bearing lubrication. Tribol Int 2012;55:46-58.

[19] Glienicke J. Experimental investigation of the stiffness and damping coefficients of turbine bearings and their application to instability prediction. Proc Inst Mech Eng 1966;181(2):116-129.

[20] Childs D, Hale K. A test apparatus and facility to identify the rotordynamic coefficients of highspeed hydrostatic bearings. ASME J Tribol 1994;116(2):337-343.

[21] Zhao SX, Zhou H, Meng G, Zhu J. Experimental identification of linear oil-film coefficients using least-mean-square method in time domain. J Sound Vibrat 2005;287:809-825.

[22] Wilkes JC, Childs DW. Improving tilting-pad journal bearing predictions-Part II: Comparison of measured and predicted rotor-pad transfer functions for a rocker-pivot tilting-pad journal bearing. ASME J Eng Gas Turbines Power 2012;135(1):012503-012511. 
[23] De Santiago O, San Andrés L. Experimental identification of bearing dynamic force coefficients in a flexible rotor. Further developments. Tribol Trans 2007;50(1):114-126.

[24] Salazar JG, Santos IF. Experimental identification of dynamic coefficients of lightly loaded tilting-pad bearings under several lubrication regimes. Inst Mech Eng Proc Part J: J Eng Tribol 2016;230(12):1423-1438.

[25] Tiwari R, Lees AW, Friswell MI. Identification of dynamic bearing parameters: A review. The Shock and Vibration Digest 2004; 36(2):99-124.

[26] Narendiranath Babu T, Manvel Raj T, Lakshmanan T. A review on application of dynamic parameters of journal bearing for vibration and condition monitoring. J Mech 2015;31(4):391416.

[27] San Andrés, L., 2010, "Notes 5.Dynamics of a rigid rotor-fluid film bearing system," Texas A\&M University Digital Libraries, https://repository.tamu.edu/handle/1969.1/93197.

[28] Tian Y, Bonis M. Analytical approach for the determination of the dynamic coefficients of hybrid bearings. Wear 1995;188:66-76.

[29] Yamamoto T, Ishida Y. Linear and nonlinear rotordynamics: A modern treatment with applications, John Wiley and Sons: New York; 2012.

[30] Lund JW. Review of the concept of dynamic coefficients for fluid film journal bearings. ASME J Tribol 1987;109:37-41.

[31] Wilkes JC, Childs DW. Improving Tilting-Pad Journal Bearing Predictions: Part I-Model Development and Impact of Rotor-Excited Versus Bearing-Excited Impedance Coefficients. ASME. Turbo Expo: Power for Land, Sea, and Air, Volume 7: Structures and Dynamics, Parts A and B 2012:965-978.

[32] Wilkes JC, Childs DW. Tilting pad journal bearings-A discussion on stability calculation, frequency dependence, and pad and pivot. J Eng Gas Turbines Power 2012;134(12):122508122517. 
Appendix A. Set of differential equations obtained by combining the expressions of the film thickness, Eq. (14), and pressure, Eq. (21), which include static and dynamic terms, with the Reynolds equation.

$\frac{1}{6} \pi h_{s}^{3} \frac{\partial^{2} p_{s}}{\partial z^{2}}+\frac{2}{3 \pi}\left(\frac{L}{D}\right)^{2} \frac{\partial}{\partial \Theta}\left(h_{s}^{3} \frac{\partial p_{s}}{\partial \Theta}\right)=O h_{s}^{3} \frac{d h_{s}}{d \Theta}$

$\frac{1}{6} \pi h_{s}^{3} \frac{\partial^{2} p_{y}}{\partial z^{2}}+\frac{2}{3 \pi}\left(\frac{L}{D}\right)^{2} \frac{\partial}{\partial \Theta}\left(h_{s}^{3} \frac{\partial p_{y}}{\partial \Theta}\right)=\left(\begin{array}{l}O \pi \sin (\pi \Theta)-\frac{3 O}{h_{s}} \cos (\pi \Theta) \frac{d h_{s}}{d \Theta}+ \\ \frac{2}{\pi} h_{s}\left(\frac{L}{D}\right)^{2} \cos (\pi \Theta) \frac{\partial p_{s}}{\partial \Theta} \frac{d h_{s}}{d \Theta}+ \\ 2 h_{s}^{2}\left(\frac{L}{D}\right)^{2} \sin (\pi \Theta) \frac{\partial p_{s}}{\partial \Theta}\end{array}\right) \Delta \eta$

$\frac{1}{6} \pi h_{s}^{3} \frac{\partial^{2} p_{\Theta}}{\partial z^{2}}+\frac{2}{3 \pi}\left(\frac{L}{D}\right)^{2} \frac{\partial}{\partial \Theta}\left(h_{s}^{3} \frac{\partial p_{\Theta}}{\partial \Theta}\right)=\left(\begin{array}{l}O \pi \cos (\pi \Theta)-\frac{3 O}{h_{s}} \sin (\pi \Theta) \frac{d h_{s}}{d \Theta}+ \\ \frac{2}{\pi} h_{s}\left(\frac{L}{D}\right)^{2} \sin (\pi \Theta) \frac{\partial p_{s}}{\partial \Theta} \frac{d h_{s}}{d \Theta}+ \\ 2 h_{s}^{2}\left(\frac{L}{D}\right)^{2} \cos (\pi \Theta) \frac{\partial p_{s}}{\partial \Theta}\end{array}\right) \eta_{s} \Delta \psi$

$\frac{1}{6} \pi h_{s}^{3} \frac{\partial^{2} p_{\dot{y}}}{\partial z^{2}}+\frac{2}{3 \pi}\left(\frac{L}{D}\right)^{2} \frac{\partial}{\partial \Theta}\left(h_{s}^{3} \frac{\partial p_{\dot{y}}}{\partial \Theta}\right)=2 O \pi \cos (\pi \Theta) \Delta \dot{\eta}$

$\frac{1}{6} \pi h_{s}^{3} \frac{\partial^{2} p_{\Theta}}{\partial z^{2}}+\frac{2}{3 \pi}\left(\frac{L}{D}\right)^{2} \frac{\partial}{\partial \Theta}\left(h_{s}^{3} \frac{\partial p_{\dot{\Theta}}}{\partial \Theta}\right)=2 O \pi \sin (\pi \dot{\Theta}) \eta_{s} \Delta \dot{\psi}$ 
Appendix B. Zero- and first-order terms of the five contributions to the pressure (static and dynamic ones) calculated using the proposed perturbation methodology (in these expressions, $h_{s}^{\prime}=\frac{d h_{s}}{d \Theta}, h_{s}^{\prime \prime}=\frac{d^{2} h_{s}}{d \Theta^{2}}$, and $h_{s}^{\prime \prime \prime}=\frac{d^{3} h_{s}}{d \Theta^{3}}$

$$
\begin{aligned}
& p_{s 0}=\frac{3}{4} \frac{O_{0} h_{s}^{\prime}\left(4 z^{2}-1\right)}{h_{s}^{3} \pi} \\
& p_{s 1}=-\frac{1}{16 \pi^{3} h_{s}^{5}}\left[-96 O_{0} h_{s} h_{s}^{\prime} h_{s}^{\prime \prime} z^{4}+48 O_{0}\left(h_{s}^{\prime}\right)^{3} z^{4}+144 O_{0} h_{s} h_{s}^{\prime} h_{s}^{\prime \prime} z^{2}-\right. \\
& -72 O_{0}\left(h_{s}^{\prime}\right)^{3} z^{2}+16 O_{0} h_{s}^{2} h_{s}^{\prime \prime \prime} z^{4}-24 O_{0} h_{s}^{2} h_{s}^{\prime \prime \prime} z^{2}-48 \pi^{2} O_{1} h_{s}^{2} h_{s}^{\prime} z^{2}- \\
& \left.-30 O_{0} h_{s} h_{s}^{\prime} h_{s}^{\prime \prime}+15 O_{0}\left(h_{s}^{\prime}\right)^{3}+5 O_{0} h_{s}^{2} h_{s}^{\prime \prime \prime}+12 \pi^{2} O_{1} h_{s}^{2} h_{s}^{\prime}\right] \\
& p_{\Theta 0}=\frac{3}{4} \frac{O_{0}\left(\cos (\pi \Theta) \pi h_{s}-3 \sin (\pi \Theta) h_{s}^{\prime}\right)\left(4 z^{2}-1\right)}{h_{s}^{4} \pi} \\
& p_{\Theta 1}=-\frac{1}{16 \pi^{3} h_{s}^{6}}\left[75 O_{0} \sin (\pi \Theta)\left(h_{s}^{\prime}\right)^{3}+144 \pi^{2} O_{0} \sin (\pi \Theta) h_{s}^{2} h_{s}^{\prime} z^{2}+\right. \\
& +216 \pi O_{0} \cos (\pi \Theta) h_{s}\left(h_{s}^{\prime}\right)^{2} z^{2}-12 \pi^{3} O_{1} \cos (\pi \Theta) h_{s}^{3}+5 \pi^{3} O_{0} \cos (\pi \Theta) h_{s}^{3}- \\
& -360 O_{0} \sin (\pi \Theta)\left(h_{s}^{\prime}\right)^{3} z^{2}-30 \pi^{2} O_{0} \sin (\pi \Theta) h_{s}^{\prime} h_{s}^{2}-45 \pi O_{0} \cos (\pi \Theta) h_{s}\left(h_{s}^{\prime}\right)^{2}+ \\
& +36 \pi^{2} O_{1} \sin (\pi \Theta) h_{s}{ }^{2} h_{s}^{\prime}+15 O_{0} \sin (\pi \Theta) h_{s}^{2} h_{s}^{\prime \prime \prime}-144 \pi O_{0} \cos (\pi \Theta) h_{s}\left(h_{s}^{\prime}\right)^{2} z^{4}- \\
& -96 \pi^{2} O_{0} \sin (\pi \Theta) h_{s}{ }^{2} h_{s}^{\prime} z^{4}-144 \pi^{2} O_{1} \sin (\pi \Theta) h_{s}{ }^{2} h_{s}^{\prime} z^{2}+16 \pi^{3} O_{0} \cos (\pi \Theta) h_{s}^{3} z^{4}+ \\
& +48 \pi^{3} O_{1} \cos (\pi \Theta) h_{s}^{3} z^{2}+576 O_{0} \sin (\pi \Theta) h_{s} h_{s}^{\prime} h_{s}^{\prime \prime} z^{2}-144 \pi O_{0} \cos (\pi \Theta) h_{s}{ }^{2} h_{s}^{\prime \prime} z^{2}- \\
& -24 \pi^{3} O_{0} \cos (\pi \Theta) h_{s}^{3} z^{2}-384 O_{0} \sin (\pi \Theta) h_{s} h_{s}^{\prime} h_{s}^{\prime \prime} z^{4}+96 \pi O_{0} \cos (\pi \Theta) h_{s}^{2} h_{s}^{\prime \prime} z^{4}+ \\
& +30 \pi O_{0} \cos (\pi \Theta) h_{s}{ }^{2} h_{s}^{\prime \prime}-120 O_{0} \sin (\pi \Theta) h_{s} h_{s}^{\prime} h_{s}^{\prime \prime}-72 O_{0} \sin (\pi \Theta) h_{s}{ }^{2} h_{s}^{\prime \prime \prime} z^{2}+ \\
& \left.+48 O_{0} \sin (\pi \Theta) h_{s}{ }^{2} h_{s}^{\prime \prime \prime} z^{4}+240 O_{0} \sin (\pi \Theta)\left(h_{s}^{\prime}\right)^{3} z^{4}\right]
\end{aligned}
$$$$
p_{y 0}=-\frac{3}{4} \frac{O_{0}\left(\sin (\pi \Theta) \pi h_{s}+3 \cos (\pi \Theta) h_{s}^{\prime}\right)\left(4 z^{2}-1\right)}{h_{s}^{4} \pi}
$$ 


$$
\begin{aligned}
& p_{y 1}=-\frac{1}{16 \pi^{3} h_{s}^{6}}\left[144 \pi^{2} O_{0} \cos (\pi \Theta) h_{s}^{2} h_{s}^{\prime} z^{2}-216 \pi O_{0} \sin (\pi \Theta) h_{s}\left(h_{s}^{\prime}\right)^{2} z^{2}-\right. \\
& -16 \pi^{3} O_{0} \sin (\pi \Theta) h_{s}^{3} z^{4}-48 \pi^{3} O_{1} \sin (\pi \Theta) h_{s}^{3} z^{2}-360 O_{0} \cos (\pi \Theta)\left(h_{s}^{\prime}\right)^{3} z^{2}+ \\
& +12 \pi^{3} O_{1} \sin (\pi \Theta) h_{s}^{3}-5 \pi^{3} O_{0} \sin (\pi \Theta) h_{s}^{3}-96 \pi^{2} O_{0} \sin (\pi \Theta) h_{s}{ }^{2} h_{s}^{\prime} z^{4}+ \\
& +240 O_{0} \cos (\pi \Theta)\left(h_{s}^{\prime}\right)^{3} z^{4}-72 O_{0} \cos (\pi \Theta) h_{s}^{2} h_{s}^{\prime \prime \prime} z^{2}+48 O_{0} \cos (\pi \Theta) h_{s}^{2} h_{s}^{\prime \prime \prime} z^{4}- \\
& -30 \pi O_{0} \sin (\pi \Theta) h_{s}{ }^{2} h_{s}^{\prime \prime}-120 O_{0} \cos (\pi \Theta) h_{s} h_{s}^{\prime} h_{s}^{\prime \prime}-30 \pi^{2} O_{0} \cos (\pi \Theta)\left(h_{s}\right)^{2} h_{s}^{\prime}+ \\
& +45 \pi O_{0} \sin (\pi \Theta) h_{s}\left(h_{s}^{\prime}\right)^{2}+24 \pi^{3} O_{0} \sin (\pi \Theta) h_{s}{ }^{3} z^{2}+36 \pi^{2} O_{1} \cos (\pi \Theta)\left(h_{s}\right)^{2} h_{s}^{\prime}+ \\
& +15 O_{0} \cos (\pi \Theta) h_{s}^{2} h_{s}^{\prime \prime \prime}+576 O_{0} \cos (\pi \Theta) h_{s} h_{s}^{\prime} h_{s}^{\prime \prime} z^{2}+144 \pi O_{0} \sin (\pi \Theta) h_{s}^{2} h_{s}^{\prime \prime} z^{2} \\
& -96 \pi O_{0} \sin (\pi \Theta) h_{s}^{2} h_{s}^{\prime \prime} z^{4}-384 O_{0} \cos (\pi \Theta) h_{s} h_{s}^{\prime} h_{s}^{\prime \prime} z^{4}+75 O_{0} \cos (\pi \Theta)\left(h_{s}^{\prime}\right)^{3}+ \\
& \left.+144 \pi O_{0} \sin (\pi \Theta) h_{s}\left(h_{s}^{\prime}\right)^{2} z^{4}-144 \pi^{2} O_{1} \cos (\pi \Theta) h_{s}{ }^{2} h_{s}^{\prime} z^{2}\right] \\
& p_{\dot{y} 0}=\frac{3}{2} \frac{O_{0} \cos (\pi \Theta)\left(4 z^{2}-1\right)}{h_{s}^{3}} \\
& \text { (B7) } \\
& p_{\dot{y} 1}=\frac{1}{8 \pi^{2} h_{s}^{5}}\left[-48 \pi O_{0} \sin (\pi \Theta) h_{s} h_{s}^{\prime} z^{4}-48 O_{0} \cos (\pi \Theta)\left(h_{s}^{\prime}\right)^{2} z^{4}+\right. \\
& +72 \pi O_{0} \sin (\pi \Theta) h_{s} h_{s}^{\prime} z^{2}+72 O_{0} \cos (\pi \Theta)\left(h_{s}^{\prime}\right)^{2} z^{2}-15 O_{0} \cos (\pi \Theta)\left(h_{s}^{\prime}\right)^{2}+ \\
& +16 \pi^{2} O_{0} \cos (\pi \Theta) h_{s}^{2} z^{4}+48 O_{0} \cos (\pi \Theta) h_{s} h_{s}^{\prime \prime} z^{4}-24 \pi^{2} O_{0} \cos (\pi \Theta) h_{s}^{2} z^{2}- \\
& -72 O_{0} \cos (\pi \Theta) h_{s} h_{s}^{\prime \prime} z^{2}+48 \pi^{2} O_{1} \cos (\pi \Theta) h_{s}{ }^{2} z^{2}-15 \pi O_{0} \sin (\pi \Theta) h_{s} h_{s}^{\prime}+ \\
& \left.+5 \pi^{2} O_{0} \cos (\pi \Theta) h_{s}{ }^{2}+15 O_{0} \cos (\pi \Theta) h_{s} h_{s}^{\prime \prime}-12 \pi^{2} O_{1} \cos (\pi \Theta) h_{s}{ }^{2}\right] \\
& p_{\Theta 0}=\frac{3}{2} \frac{O_{0} \sin (\pi \Theta)\left(4 z^{2}-1\right)}{h_{s}^{3}} \\
& p_{\dot{\Theta} 1}=-\frac{1}{8 \pi^{2} h_{s}^{5}}\left[-48 \pi O_{0} \cos (\pi \Theta) h_{s} h_{s}^{\prime} z^{4}+48 O_{0} \sin (\pi \Theta)\left(h_{s}^{\prime}\right)^{2} z^{4}+\right. \\
& +72 \pi O_{0} \cos (\pi \Theta) h_{s} h_{s}^{\prime} z^{2}-72 O_{0} \sin (\pi \Theta)\left(h_{s}^{\prime}\right)^{2} z^{2}+16 \pi^{2} O_{0} \sin (\pi \Theta) h_{s}^{2} z^{4}- \\
& -48 O_{0} \cos (\pi \Theta) h_{s} h_{s}^{\prime \prime} z^{4}+24 O_{0} \pi^{2} \sin (\pi \Theta) h_{s}^{2} z^{2}+72 O_{0} \sin (\pi \Theta) h_{s} h_{s}^{\prime \prime} z^{2}- \\
& -48 \pi^{2} O_{1} \sin (\pi \Theta) h_{s}^{2} z^{2}-15 \pi O_{0} \cos (\pi \Theta) h_{s} h_{s}^{\prime}+15 O_{0} \sin (\pi \Theta)\left(h_{s}^{\prime}\right)^{2}- \\
& \left.-5 \pi^{2} O_{0} \sin (\pi \Theta) h_{s}^{2}+15 O_{0} \sin (\pi \Theta) h_{s} h_{s}^{\prime \prime}-12 \pi^{2} O_{1} \sin (\pi \Theta) h_{s}^{2}\right]
\end{aligned}
$$




\section{List of Figures}

Figure 1. Geometry notation and coordinate systems (left). Representation in Cartesian coordinates (right).

Figure 2. Representation, using dimensionless variables, of the location of bearing and journal centers when vibrating in the vicinity of the static equilibrium point.

Figure 3. Schematic representation of the fluid-film dynamic coefficients according to a mechanical model of springs and dashpots.

Figure 4. Ocvirk number as a function of eccentricity ratio for different aspect ratios. Exact (Num) and approximate (P\&O) solutions compared to the Ocvirk solution for infinitely short JB (ISJB).

Figure 5. Dimensionless pressure profiles predicted at $z=0$ for $L / D=0.75$ and $\eta=0.5$.

Figure 6. Dimensionless pressure profiles at $z=0$ and $\Theta=0.5$ as a function of aspect ratio (left, $\eta=0.5$ ) and eccentricity (right, $L / D=0.5$ ).

Figure 7. Dimensionless friction coefficient as a function of eccentricity for different aspect ratios.

Figure 8. Dimensionless axial flow rate as a function of eccentricity for different aspect ratios.

Figure 9. Dimensionless phase angle as a function of eccentricity for different aspect ratios.

Figure 10. Dimensionless stiffness coefficients $k_{\underline{x x}}$ and $k_{\underline{y x}}$ as a function of eccentricity for different aspect ratios.

Figure 11. Dimensionless stiffness coefficients $k_{\underline{y y}}$ and $k_{\underline{x y}}$ as a function of eccentricity for different aspect ratios.

Figure 12. Dimensionless damping coefficients $c_{\underline{x x}}$ and $c_{\underline{y x}}$ as a function of eccentricity for different aspect ratios.

Figure 13. Dimensionless damping coefficients $c_{y \underline{y}}$ and $c_{\underline{x y}}$ as a function of eccentricity for different aspect ratios.

Figure 14. Global error of the ISJB approximation and the P\&O-perturbation in the calculation of the dynamic coefficients, calculated using Eq. (46). 
Figure 15. Dynamic coefficients (absolute values) as a function of Ocvirk number.

Comparison of experimental and theoretical data from Zhao et al. [21] for $\mathrm{L} / \mathrm{D}=0.58$ with predictions of the $\mathrm{P} \& \mathrm{O}$ model. The grey ovals indicate the dispersion in the experimental data.

Graphical abstract

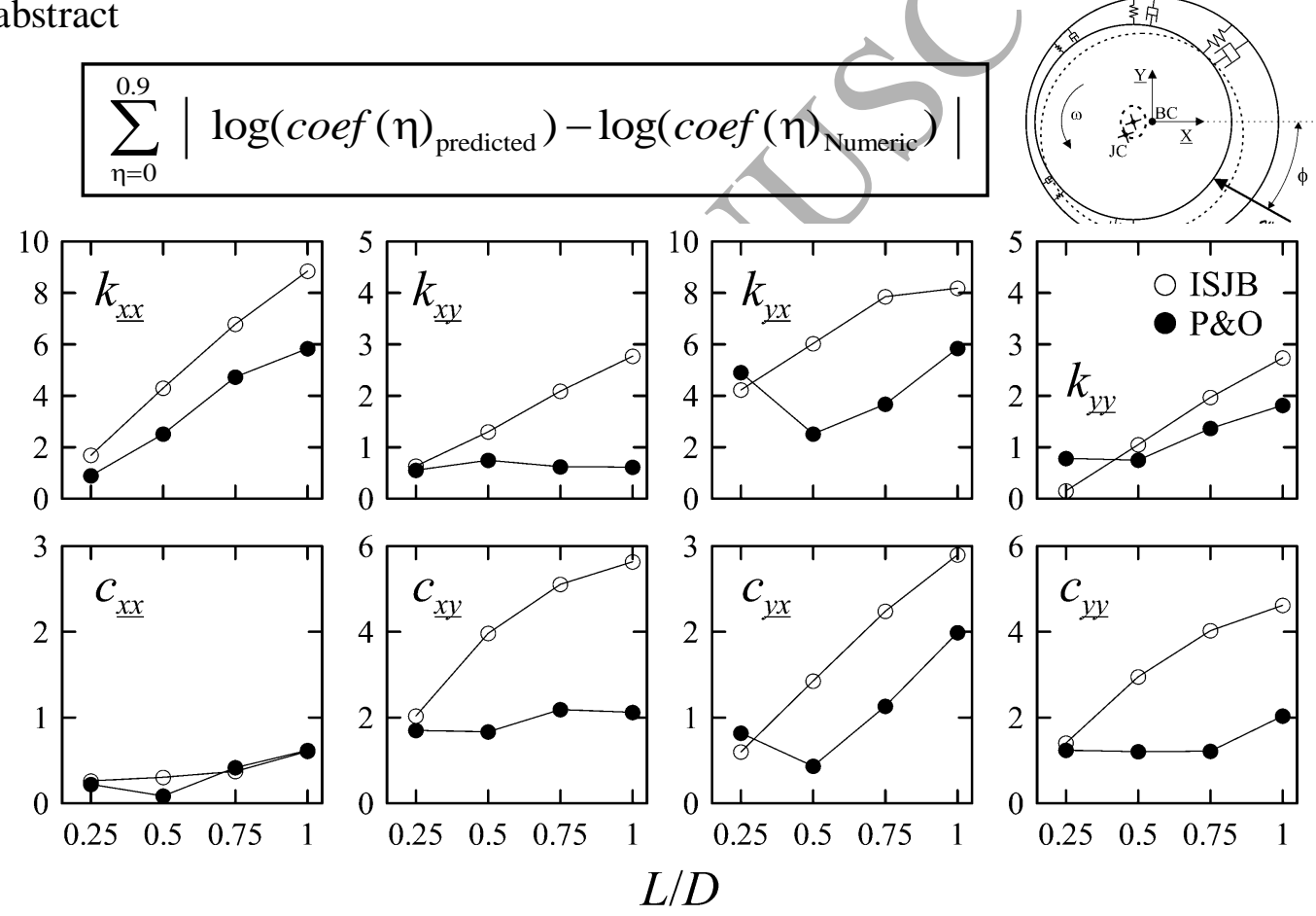

